\title{
Article \\ The Automatic Design of Multimode Resonator Topology with Evolutionary Algorithms
}

\author{
Vladimir V. Stanovov ${ }^{1,2}\left(\mathbb{D}\right.$, Sergey A. Khodenkov ${ }^{1}\left(\mathbb{D}\right.$, Aleksey M. Popov ${ }^{1}$ and Lev A. Kazakovtsev ${ }^{1,3, * \mathbb{C}}$ \\ 1 Institute of Informatics and Telecommunications, Reshetnev Siberian State University of Science and \\ Technology, 31 Krasnoyarsky Rabochy av., 660037 Krasnoyarsk, Russia; vladimirstanovov@yandex.ru (V.V.S.); \\ hodenkov@sibsau.ru (S.A.K.); vm_popov@sibsau.ru (A.M.P.) \\ 2 Institute of Space and Information Technologies, Siberian Federal University, 79 Svobodny pr., \\ 660041 Krasnoyarsk, Russia \\ 3 Institute of Business Process Management, Siberian Federal University, 79 Svobodny pr., \\ 660041 Krasnoyarsk, Russia \\ * Correspondence: levk@bk.ru
}

check for updates

Citation: Stanovov, V. V.; Khodenkov, S. A.; Popov, A. M.; Kazakovtsev, L.

A. The Automatic Design of Multimode Resonator Topology with Evolutionary Algorithms. Sensors 2022, 22, 1961. https:// doi.org/10.3390/s22051961

Academic Editor: Massimo Donelli

Received: 29 January 2022

Accepted: 26 February 2022

Published: 2 March 2022

Publisher's Note: MDPI stays neutral with regard to jurisdictional claims in published maps and institutional affiliations.

Copyright: (C) 2022 by the authors. Licensee MDPI, Basel, Switzerland. This article is an open access article distributed under the terms and conditions of the Creative Commons Attribution (CC BY) license (https:// creativecommons.org/licenses/by/ $4.0 /$ )

\begin{abstract}
Microwave electromagnetic devices have been used for many applications in tropospheric communication, navigation, radar systems, and measurement. The development of the signal preprocessing units including frequency-selective devices (bandpass filters) determines the reliability and usability of such systems. In wireless sensor network nodes, filters with microstrip resonators are widely used to improve the out-of-band suppression and frequency selectivity. Filters based on multimode microstrip resonators have an order that determines their frequency-selective properties, which is a multiple of the number of resonators. That enables us to reduce the size of systems without deteriorating their selective properties. Various microstrip multimode resonator topologies can be used for both filters and microwave sensors, however, the quality criteria for them may differ. The development of every resonator topology is time consuming. We propose a technique for the automatic generation of the resonator topology with required frequency characteristics based on the use of evolutionary algorithms. The topology is encoded into a set of real valued parameters, which are varied to achieve the desired features. The differential evolution algorithm and the genetic algorithm with simulated binary crossover and polynomial mutation are applied to solve the formulated problem using the dynamic penalties method. The experimental results show that our technique enables us to find microstrip resonator topologies with desired amplitude-frequency characteristics automatically, and manufactured devices demonstrate characteristics very close to the results of the algorithm. The proposed algorithmic approach may be used for automatically exploring the new perspective topologies of resonators used in microwave filters, radar antennas or sensors, in accordance with the defined criteria and constraints.
\end{abstract}

Keywords: multimode resonator; amplitude-frequency characteristics; microwave sensor; optimization; differential evolution; parameter adaptation

\section{Introduction}

Microstrip resonators are traditionally used to build on their basis high-frequency frequency-selective devices with high-quality electrical characteristics, which are distinguished by manufacturability and low cost in mass production [1]. In addition, they are used in microstrip devices for measuring the electrical characteristics of various materials and substances, that is, they can be used as microwave sensor designs [2-4].

The rapid development of telecommunication technologies for the needs of tropospheric and space communications, radar and navigation systems [5], unmanned vehicles [2] is accompanied by a constant increase in the frequency-selective properties of microwave devices, such as bandpass filters, high and low pass filters, diplexers [6,7]. 
Such microwave bandpass filters [5,8-10] are also traditionally used in radio equipment. However, the development of these promising designs implies not only a constant improvement in their electrical characteristics and a reduction in size, but also a significant expansion of the operating bandwidth, which, accordingly, can significantly expand information transmission channels, or, on the contrary, cut off interference in a wide range. Therefore, the study of multimode microstrip resonators is an urgent and important task. Using the design features of a microstrip conductor, it is possible to significantly bring together several of its lowest oscillation modes in frequencies, which will form a wide bandwidth. It is important that, in terms of such parameters as miniaturization, reliability, manufacturability, and cost, devices based on such resonators [11], including ultra-wideband (UWB) ones, are among the best.

On the basis of microstrip resonators, it is possible to effectively implement both bandpass filters and notch filters (stop band filters) $[12,13]$. At the same time, the scope of microstrip resonators is not limited to frequency-selective designs. Microwave sensors designed on their basis are of high interest [14-16]. The concept of microwave sensors based on microstrip resonators is based on changes in their frequency response depending on the dielectric permittivity of the samples under test which are considered as an additional dielectric layer [14,17-21] or are introduced in the cleft of the proposed sensor [16,22].

Microwave sensors based on microstrip resonators [23] are used for measuring complex permittivity of materials, dielectric measurements of solids and liquids [24-26], gas sensing [15], non-invasive medical measurements [26-29] and other applications [30-32]. Permittivirt measurement methods of dielectric materials available in sheet, liquid, paste, powder, etc., forms by microwave resonators used as sensors were summarized in $[33,34]$.

Moreover, the rapid development of the Internet of Things and sensor networks requires many miniature devices to work together in a limited frequency range without mutual interference [35]. Microstrip filter is an important component of wireless sensor network nodes, which selects useful signals and suppresses clutter interference signals. Multi-mode microstrip filters are required to prevent signal crosstalk between adjacent channels [36]. This requires high frequency selectivity and out-of-band rejection of the filters.

Particularly noteworthy are the designs of bandpass filters based on two-mode [1,22], three-mode [37] and multimode resonators. In the formation of the passband of such devices, in contrast to one-mode microstrip filters, the resonances of multiple lowest oscillation modes from each resonator are involved. As a result, the order of the filter multiplies, which is known to determine its selectivity. Due to the small number of links in such filters, not only the overall dimensions are significantly reduced in comparison with traditional designs, but the losses in the passband are also reduced. Strip conductors of multi-mode resonators in a microstrip filter are usually in the form of a square, a square or rectangular frame, sometimes they are made in a form of a closed meander line, which also has a fourth-order symmetry axis [5]. The natural frequencies of the first two oscillation modes in such resonators are degenerate, and the spatial distributions of the amplitudes of high-frequency fields are orthogonal. In this case, in a bandpass filter, the required amount of coupling between the resonances of two oscillation modes is provided either by etching one of the corners of a conductor in the shape of a square or square frame, or by adding a segment of a slotted line or a strip coupling element, which, as a rule, are located at an angle of $45^{\circ}$ to the resonator axes [1].

Two-mode resonators with a regular strip conductor partially split at one end by a narrow slit [38] are more miniature. In this case, the value of the link between the first two modes of resonator oscillations is small, and it is implemented by a small difference in the length of the conductors in its split section. This circumstance does not allow us to develop the broadband filters based on such resonators; however, filters with a narrow passband on them have sufficiently high frequency-selective properties even in designs consisting of only two or three resonators [39].

Such frequency-selective devices allow us to implement them in multiple operation modes when a certain form of their strip conductors is set. As a result, the natural fre- 
quencies of several lowest oscillation modes could be brought together and miniaturize the structure [40].

Requirements for resonators may differ. In some cases (filters, radar antennas, etc.), the topology is required to cover a wide range of frequencies. At the same time, the prospects for designing sensor elements based on multimode microstrip resonators may be due to the ability to cover a wide range of interference when measuring the dielectric properties of substances. Specific types of sensors (e.g., gas sensors) require the use of a resonator with a very narrow bandwidth. Nevertheless, the problem of searching for a resonator design with a given center frequency, as well as with a given bandwidth, considered in this paper, is relevant for most types of devices

Due to their small circuit sizes (up to several millimeters in each dimension), such resonators can be used in hybrid integration with small wireless networking and radar devices [41,42]. Ultra-wideband (multi-mode) microwave filters are an essential part of the radar sensor systems $[4,43,44]$ and antenna sensors [2] which are of high interest due to the development of unmanned vehicles and robotics. Such systems include both microstrip resonators with the simplest geometric configuration [45-47] and more complex designs [48,49].

For the sensor network nodes, it is essential that the central frequency points of the filter has a certain capacity bandwidth $[35,50]$.

We consider the problem of computer-aided design of microstrip structures of multimode resonators with the strip conductors in the form of a combination of rectangles $[1,37]$. Filters based on such multi-mode resonators are implemented not only on substrates with a high dielectric constant, but also with a low one [1]. Such designs, due to the attenuation poles existing near the passband, are distinguished by an increased steepness of the amplitude-frequency characteristic (AFC) slopes, wide barrage bands and a high level of microwave power suppression in them which is essential for their application in sensor network nodes.

Well-known standard mathematical optimization methods such as the random search method, the Davidon-Fletcher-Powell algorithm, the similex Nelder-Mead method and genetic algorithms are implemented as subprograms in all popular software products for the design of microwave devices. An experienced user of these products can successfully optimize single mode resonator filters. Some promising results have been achieved in the development of methods for optimizing the designs of two-mode resonators [51]. Since it was stated that standard methods of mathematical programming were unsuitable even for the optimization of two-mode constructions, for the development of such devices, special optimization methods based on taking into account the physical principles and features of each specific design were proposed, thus not being in any way universal. In the computer-aided development of such systems, expert systems are widely used.

Modern software products designed for the numeric electrodynamic analysis of microwave device structures make it possible to simulate the amplitude-frequency response of a microstrip resonators of various types with high accuracy. The capabilities of modern computing systems enables us to perform electrodynamic analysis many times, while changing the geometric parameters of the microwave device. Electrodynamic analysis software products provide an application programming interface through which an external program can set the required device configuration.

The concept of this work is to develop evolutionary methods for optimizing the designs of microwave microstrip resonators including multimode ones, in which the well-known methods of electrodynamic analysis, implemented in modern software products, are used to assess the AFC, which, in turn, is used to calculate the fitness function in the evolutionary algorithms.

The recent advances in the area of optimization methods that are capable of solving a variety of problems, such as evolutionary algorithms (EAs) [52], open new possibilities for automation of many routines, including those present in the area of microwave filters design. In particular, the existing EAs, such as genetic algorithms (GAs) [53], particle 
swarm optimization (PSO) [54], differential evolution (DE) [55] and many others [56] do not require specific information about the type of the problem to be solved or information about the derivative of the fitness function. Nevertheless, when combined with certain constraint handling techniques, they enable us to efficiently solve many problems, which arise in industrial applications [57].

This study is focused on the application of one of the variants of DE, namely the LSHADE algorithm [58] with several modifications, including mutation strategy, crossover operators and constraint handling technique, to the problem of designing the microstrip resonator topology. For this purpose, we propose an encoding method, allowing different topologies to be tested, and the specific settings for the optimization algorithm to be set.

The rest of the paper is organized as follows. The second section describes the methods used in our research and includes a description microstrip microwave resonators and their properties, description of the existing DE algorithm version and other real-coded evolutionary algorithms, and the proposed approach to the automated microwave device design. The experimental setup and results are demonstrated in the third section, and the last section concludes this study.

\section{Materials and Methods}

\subsection{Microstrip Microwave Resonators}

When calculating the amplitude-frequency characteristics (AFC) of all the considered resonators, we used monolithic substrates made of materials widely used in microwave technology (TBNS and Policor) with the same plate thickness $h=1 \mathrm{~mm}$ but different relative permittivity $\varepsilon=80$ and $\varepsilon=9.8$, respectively.

The microwave resonators are characterized by certain criteria, which are functionals of the AFC. In this study, we propose an approach to the automated design of the resonators and implement it for three types of devices, namely the U-shaped, U-shaped with an additional cut and $\mathrm{W}$-shaped resonators. The strip conductors of the resonators have the following geometric shapes: slot-split rectangular (U-shaped), twice slot-split rectangular (U-shaped with an additional cut), and studs with a plume (W-shaped), respectively. However, our approach does not depend on the shape of a flat microstrip resonator and can be implemented to the resonators of various shapes.

The bandwidth of the first considered resonator type (Figure 1) can be formed both by its two lowest oscillation modes [38] and by three ones (Figure 2) [39]. Changing the width and depth of the cut in the conductor makes it possible to bring these lower natural frequencies closer together in such a resonator. In this case, the relative bandwidth $d F=\Delta F / F_{0}$ of such a two-mode resonator does not exceed $40 \%$, where $\Delta F$ is the bandwidth measured at the level of $-3 \mathrm{~dB}$ from the level of the minimum loss in the bandwidth, $F_{0}$ is the center frequency of the bandwidth. For the design of devices with a wide frequency range, a three-mode resonator is more promising. Our studies using Policor dielectric plates, at a fixed central frequency of the resonator $F_{0}=3 \mathrm{GHz}$ show that its minimum bandwidth is $45 \%$, and the maximum bandwidh is $71 \%$. The first resonator was set to operate in a threeresonance mode [39], its structure is shown in Figure 1. Such a U-shaped resonator is a very simple construction which is one of the most widely used in various microwave devices including sensors [3,59-62]. Depending on the dimensions, such devices have various AFCs and purposes. Similar forms such as Y-shaped resonators are also popular [63]. The theoretically calculated data for a three-mode resonator implemented on a TBNS dielectric plate, obtained using a numerical electrodynamic analysis of its 3D model, are in almost ideal agreement with the data taken on an actually manufactured prototype [39]. 

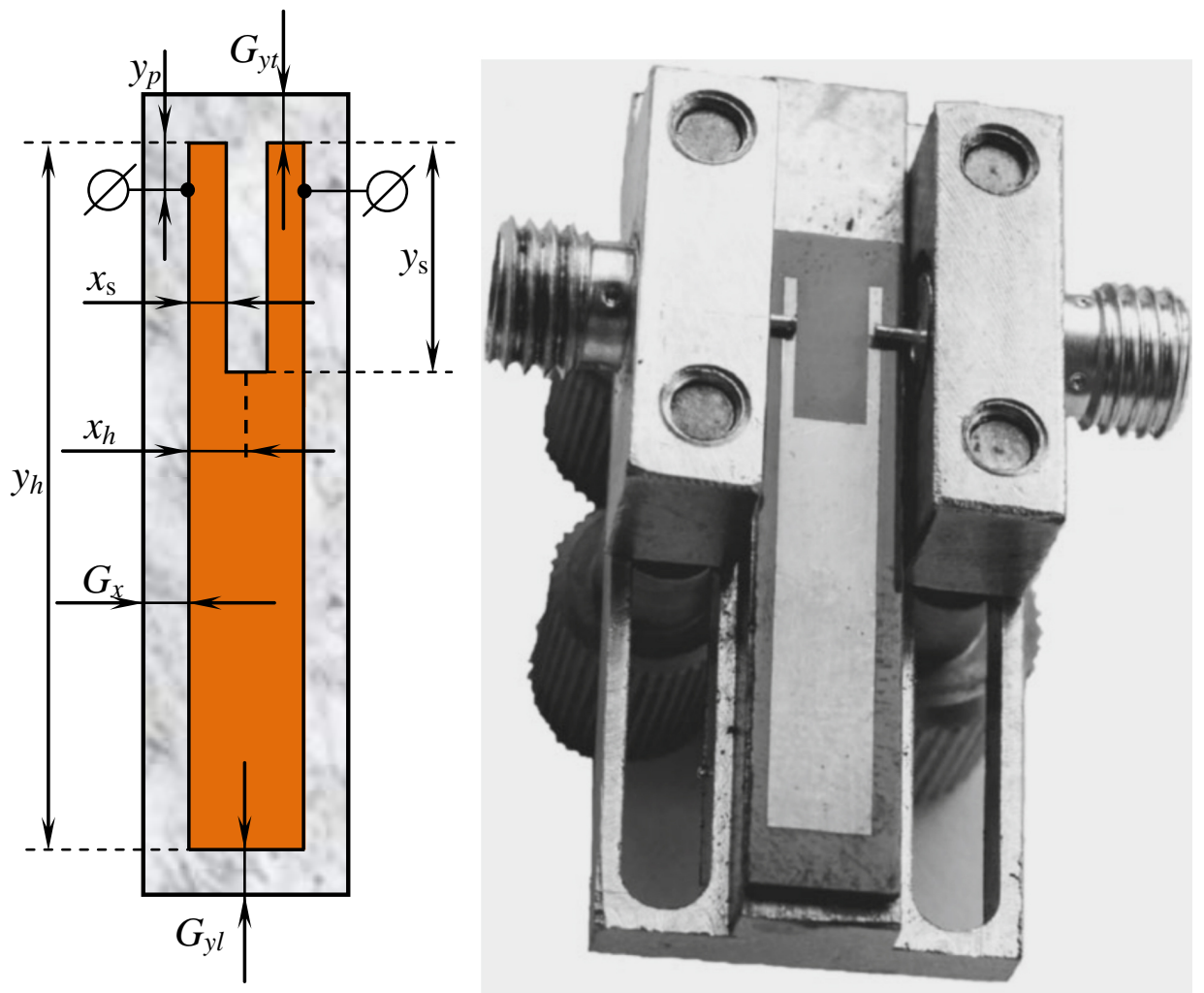

Figure 1. U-shaped (slot-split rectangular) 3-mode resonator: layout and manufactured device.

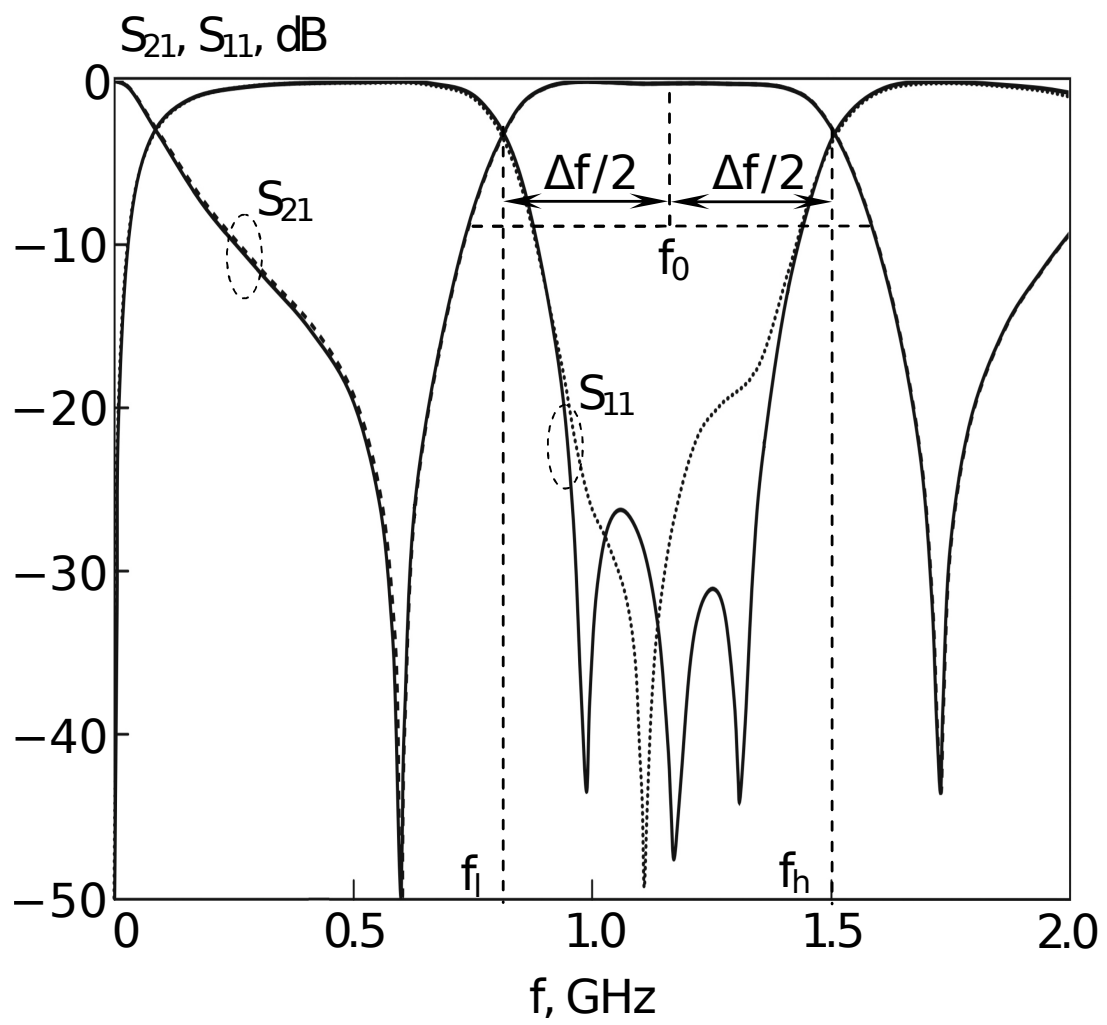

Figure 2. AFC of the U-shaped 3-mode (slot-split rectangular) resonator. Points-experimental data, lines-theoretical data (results of electrodynamic modelling).

When splitting the strip conductor of the MPR by a narrow slit [38], it is possible to bring the natural frequencies of its first two oscillation modes closer together. This allows such two-mode resonators to create not only band-pass filters with high frequency-selective 
properties, but also two-band filters, as well as diplexers. However, the relative bandwidth of such filters does not exceed $20 \%$, while the investigated resonators make it possible to develop filters with bandwidths up to $70 \%$, and such devices have high frequencyselective properties.

In addition, Figure 1 shows the main geometrical parameters required to encode such a resonator for the optimization algorithm. The top and the lower gaps are $G_{y t}$ and $G_{y l}$, respectively, and the horizontal gap is $G_{x}$, which is the same on both sides. Mentioned gaps are fixed during experiments: if the resonator size changes, then the enclosure size also changes to keep the same gaps. The resonator has the following geometrical properties:

1. $x_{h}$-half of resonator width, in mm (only one side is encoded and then mirrored);

2. $y_{h}$-resonat's height, in $\mathrm{mm}$;

3. $x_{s}$-relative width of the upper part, $x_{s} \in[0,1]$, i.e., if $x_{s}=1$ then the width is equal to $x_{h}$;

4. $y_{s}$-relative height of the upper part, $y_{s} \in[0,1]$, i.e., if $y_{s}=0$ then the height is equal to 0 (no central cut);

5. $y_{p}$-relative height of the position of the side conductors to ports, $y_{p} \in[0,1]$, i.e., if $y_{p}=0$, then the conductors are located at the top.

The following parameters of the resonator are shown in Figure 1: $G_{x}=35 \mathrm{~mm}$, $G_{y l}=20 \mathrm{~mm}, G_{y t}=28 \mathrm{~mm}, x_{h}=13.25 \mathrm{~mm}, y_{h}=174.16 \mathrm{~mm}, x_{s}=0.61, y_{s}=0.325$, $y_{p}=0.019$. Depending on the ratio of these parameters, devices with completely different amplitude-frequency characteristics and purposes can be obtained [3,60,62]. Figure 2 shows the results of the AFC measurement performed using the electrodynamic numerical analysis for the resonator presented in Figure 1.

Figure 2 shows the frequency dependence of the forward loss for the passage of microwave power (transmission factor S21), as well as the frequency dependence of the reflection loss of microwave power (transmission factor S11). The most important parameters that characterize the resonator are the bandwidth $\Delta F=F_{h}-F_{l}$, where $F_{h}$ and $F_{l}$ are the high-frequency and low-frequency boundaries of the bandwidth measured at the level of $-3 \mathrm{~dB}$ from the level of minimum losses in the bandwidth, and also the center frequency of the passband $F_{0}$. For the resonator in Figure 1, these parameters were as follows: $F_{0}=1.2 \mathrm{GHz}$. Therelative bandwidth $d F$, measured as $d F=\frac{F_{h}-F_{l}}{F_{0}}$ is more convenient for the use in optimization algorithm than $\Delta F$. In our case, $d F=0.7$. Both $F_{0}$ and $d F$ are functionals of the AFC.

Figure 3 shows the topology of the 6-mode microstrip resonator with a doubly slotted strip conductor. All theoretical and experimental studies for this resonator were carried out using a substrate with a relative permittivity $\varepsilon=9.8$ (Polycor). In this case, just as for a three-mode resonator implemented on a substrate with a high dielectric constant, the data for a six-mode resonator (Figuse 4) obtained by numerical electrodynamic analysis of its 3D model are in good agreement with the data taken on a really manufactured prototype. This allows us to confine ourselves in further studies of microstrip resonators to only numerical experiments, and, moreover, conduct such experiments in an automated mode under control of an optimization algorithm.

At a fixed center frequency $F_{0}=3 \mathrm{GHz}$, its minimum bandwidth is $79 \%$, and the maximum one is $81 \%$, which is significantly wider than that of a three-mode resonator. 

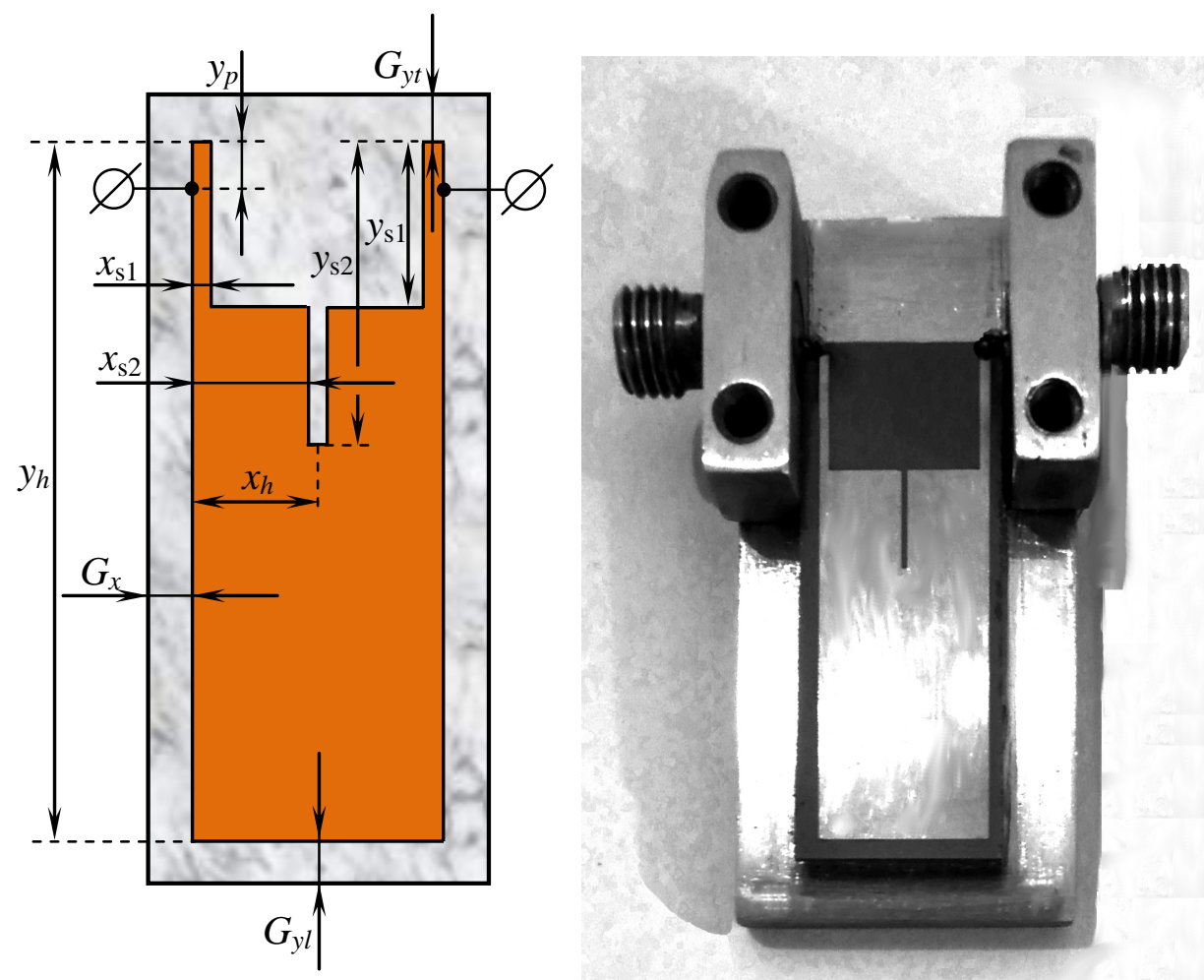

Figure 3. U-shaped (twice slot-split rectangular) 6-mode resonator with an additional cut: layout and manufactured device.

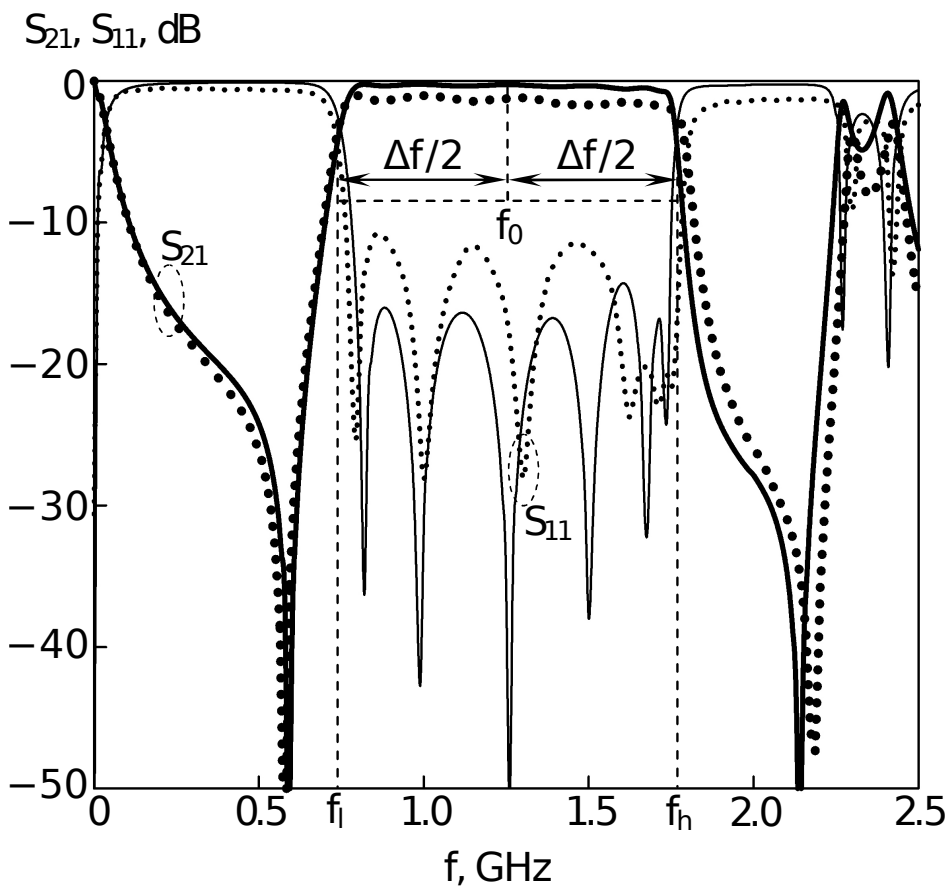

Figure 4. AFC of the U-shaped (twice slot-split rectangular) 6-mode resonator with an additional cut. Points - experimental data, lines - theoretical data (results of electrodynamic modelling).

The 6-mode resonator, considered in this study, is similar to the 3-mode one, however, it has an additional cut in the middle. The parameters used to describe this resonator are similar to those of the previous one:

1. $x_{h}$-half of resonator width, in mm (only one side is encoded and then mirrored);

2. $y_{h}$-resonator height, in $\mathrm{mm}$; 
3. $x_{s 1}$-relative width of the upper part, $x_{s 1} \in[0,1]$, i.e., if $x_{s 1}=1$ then the width is equal to $x_{h}$;

4. $y_{s 1}$-relative height of the upper part, $y_{s 1} \in[0,1]$, i.e., if $y_{s 1}=0$ then the height is equal to 0 (no central cut);

5. $x_{s 2}$-relative width of the small central cut, $x_{s 2} \in[0,1]$, i.e., if $x_{s 2}=1$ then the width is equal to $x_{h}$;

6. $y_{s 2}$-relative height of the small central cut, $y_{s 2} \in[0,1]$, i.e., if $y_{s 2}=0$ then the height is equal to 0 (no central cut);

7. $y_{p}$-relative height of the position of the side conductors to ports, $y_{p} \in[0,1]$, i.e., if $y_{p}=0$, then the conductors are located at the top.

The following parameters of the resonator are shown in Figure 3: $G_{x}=35 \mathrm{~mm}$, $G_{y l}=24 \mathrm{~mm}, G_{y t}=30 \mathrm{~mm}, x_{h}=49.5 \mathrm{~mm}, y_{h}=280 \mathrm{~mm}, x_{s 1}=0.121, y_{s 1}=0.236$, $x_{s 2}=0.969, y_{s 2}=0.204, y_{p}=0.02$. Figure 4 shows the results of the AFC measurement performed using the electrodynamic numerical analysis for the resonator presented in Figure 3. The study was carried out using a substrate with a relative permittivity $\varepsilon=9.8$.

In Figure 4, the frequency dependence of direct losses from port 1 to port 2 and frequency dependence of losses in reflection, from port 1 to port 1 , are shown. For this case, $F_{0}$ is around $1.26 \mathrm{GHz}$, and $d F=0.8$.

Figure 5 shows the topology of the 5 -mode W-shaped (studs with a plume) microstrip resonator. Variation in the length of the stripe segment of the plume is accompanied by a decrease in the frequencies of the even modes of oscillation of this resonator, while the frequencies of its odd modes remain unchanged. This makes it possible to form the passband of this resonator by its five lowest resonances, while the relative width of its passband is $91 \%$. We point out that $d F$ can be varied from $74 \%$ to $97 \%$, which allows us to recognize it as the most promising for building ultra-wideband devices, among the resonators considered in this paper.

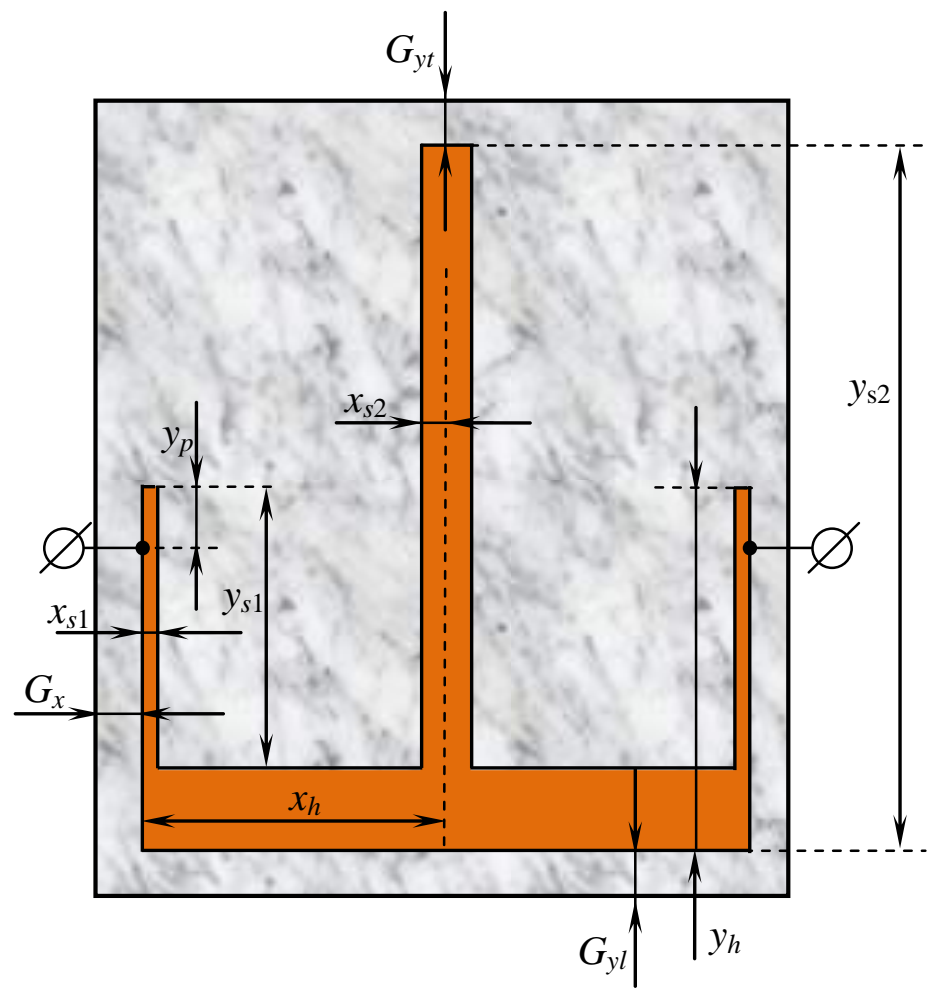

Figure 5. W-shaped (studs with a plume) 5-mode resonator: layout.

The 5-mode resonator considered in this study has a W-shaped topology. The following parameters are used to describe this resonator: 
1. $x_{h}$-half of resonator width, in mm (only one side is encoded and then mirrored);

2. $y_{h}$-resonator height (without central part), in mm;

3. $x_{s 1}$-relative width of the side part, $x_{s 1} \in[0,1]$, i.e., if $x_{s 1}=1$ then the width is equal to $x_{h}$;

4. $y_{s 1}$-relative height of the side part, $y_{s 1} \in[0,1]$, i.e., if $y_{s 1}=0$ then the height is equal to 0 ;

5. $x_{s 2}$-relative width of the small central cut, $x_{s 2} \in[0,1]$, i.e., if $x_{s 2}=1$ then the width is equal to $x_{h}$;

6. $y_{s 2}$-relative height of the small central cut, $y_{s 2} \in[0,3]$, i.e., if $y_{s 2}=0$ then the height is equal to 0 (no central cut);

7. $y_{p}$-relative height of the position of the side conductors to ports, $y_{p} \in[0,1]$, i.e., if $y_{p}=0$, then the conductors are located at the top.

Thus, the following parameters of the resonator are demonstrated Figure 5: $G_{x}=35 \mathrm{~mm}$, $G_{y l}=24.5 \mathrm{~mm}, G_{y t}=30 \mathrm{~mm}, x_{h}=78 \mathrm{~mm}, y_{h}=93.5 \mathrm{~mm}, x_{s 1}=0.0384, y_{s 1}=0.770$, $x_{s 2}=0.075, y_{s 2}=1.98, y_{p}=0.0145$. Figure 6 shows the results of the AFC measurement performed with use of electrodynamic numerical analysis for the resonator presented in Figure 5.

$$
S_{21}, S_{11}, d B
$$

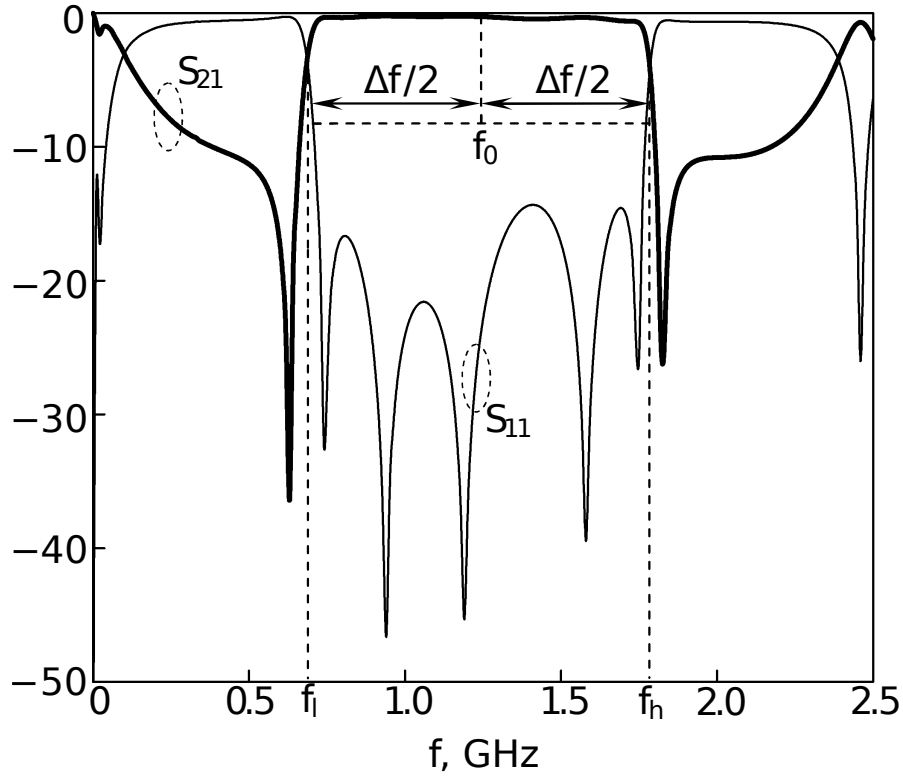

Figure 6. AFC of the W-shaped (studs with a plume) 5-mode resonator. Points - experimental data, lines - theoretical data (results of electrodynamic modelling).

The described microstrip resonators will be further considered in this study for tuning their passband frequency by changing the topology parameters. The search of optimal resonator topology will be performed with the purpose of tuning the known topology to work in a different frequency ranges: for example, the resonator shown in Figure 3 has $F_{0}=1.26 \mathrm{GHz}$ with $d F=0.8$, and the goal will be to increase the frequency to $1.5 \mathrm{GHz}$ with the same relative bandwidth $d F=0.8$. A simple scaling of the resonator geometrical properties does not give a proper result in most cases: the resulting frequency range is different from the desired as well as the width $\Delta F$. Moreover, the number of modes could be changed.

\subsection{Evolutionary Continuous Optimization Methods}

Among the existing optimization methods for continuous search spaces, the evolutionary algorithms are considered as the most efficient global optimization techniques, as long as they are capable of finding global optimum even for complicated landscapes 
due to their population-based structure and randomized search. Various evolutionary algorithms are a popular and efficient tool for microelectronic device design [64-66] as well as data processing for evaluation of their quality $[67,68]$. Therefore, in this study, two evolutionary optimizers were considered: the differential evolution algorithm and the genetic algorithm (GA). The next two subsections provide a brief description of these methods.

\subsection{Differential Evolution}

The Differential Evolution is a population-based optimization method, originally proposed by Storn and Price in [69]. The main idea of the DE approach was to use the difference vectors between individuals in the population and apply these scaled vectors to the existing points in the search space to sample new solutions. DE appeared to be very efficient and easy to implement, although it has a small number of parameters. Such algorithms are of a high demand in various areas including sensor design [70]. The algorithm starts with a set of $N P$ initial points in a $D$-dimensional search space, $x_{i, j}, i=1 \ldots N P, j=1 \ldots D$, generated using uniform distribution within $\left[x \min _{j}, x \max _{j}\right], j=1 \ldots D$. After initialization, the main loop starts, which includes mutation, crossover and selection.

There are several mutation strategies used in DE, with rand/1, used in [69], and current-to-pbest/1, proposed for the JADE [71] algorithm, being the most popular ones. The current-to-pbest/1 strategy is described with the following equation:

$$
v_{i, j}=x_{i, j}+F \cdot\left(x_{p b e s t, j}-x_{i, j}\right)+F \cdot\left(x_{r 1, j}-x_{r 2, j}\right),
$$

where pbest is an index of one of the $p \cdot 100 \%$ best individuals, $r 1$ and $r 2$ are randomly chosen indexes, and pbest $\neq r 1 \neq r 2 \neq i, F$ is the scaling factor parameter, usually in range $[0,1], v$ is the set of mutant vectors, and $x$ are called target vectors.

After the mutant vectors $v$ are generated by mutation, the crossover is performed to generate trial vectors $u$. The most used method is the binomial crossover, in which the trial vector receives coordinates from mutant vector with probability $\mathrm{Cr} \in[0,1]$. The binomial crossover could be described as follows:

$$
u_{i, j}=\left\{\begin{array}{ll}
v_{i, j}, & \text { if } \operatorname{rand}(0,1)<C r \text { or } j=j r a n d \\
x_{i, j}, & \text { otherwise }
\end{array},\right.
$$

where jrand is a randomly generated index in $[1, D]$. It is needed to make sure that at least one component of the trial vector is different from the target vector. Otherwise, the trial vector would be exactly the same as target vector, which would lead to unnecessary calculations. After the set of trial vectors is generated, the bound constraint handling $(\mathrm{BCH})$ method is applied. One of the widely used bound constraint handling methods [72] is described as follows:

$$
u_{i, j}=\operatorname{rand}\left(x \min _{j}, x \max _{j}\right) \text { if } u_{i, j} \notin\left[x \min _{j}, x \max _{j}\right] .
$$

Here, if the newly generated point is out of bounds, its parent is used to set the new position. However, in this study the following BCH approach was used:

$$
u_{i, j}=\left\{\begin{array}{ll}
\frac{x \min _{j}+x_{i, j}}{2}, & \text { if } u_{i, j}<x \min _{j} \\
\frac{\operatorname{mmax}_{j}+x_{i, j}}{2}, & \text { if } u_{i, j}>x \max _{j}
\end{array} .\right.
$$

Here, $x \min _{j}$ and $x_{\max }$ are the lower and the upper boundaries for the $j$-th variable. After the fitness values $f(u)$ are calculated, the selection step is performed:

$$
x_{i}=\left\{\begin{array}{ll}
u_{i}, & \text { if } f\left(u_{i}\right) \leq f\left(x_{i}\right) \\
x_{i}, & \text { if } f\left(u_{i}\right)>f\left(x_{i}\right)
\end{array} .\right.
$$


If the newly generated solution $u_{i}$ is not worse than its parent individual $x_{i}$ from the population, then the replacement occurs. Such a selection operator is greedy, and it allows DE to solve multimodal problems efficiently.

\subsection{L-SHADE Algorithm}

One of the main problems of the DE algorithm is its sensitivity to three main parameters: scaling factor $F$, crossover rate $C r$ and population size $N P$. Therefore, in many studies, the parameter adaptation techniques have been proposed for DE, for example, the previously mentioned JADE algorithm [71]. Based on the JADE parameter adaptation, the SHADE [73] and L-SHADE [58] approaches were developed, which appeared to be highly competitive and popular nowadays, according to recent studies [74]. Below, the LSHADE approach will be described.

The SHADE and the L-SHADE algorithms use the Success-History Adaptation (SHA) with a set of $H=5$ memory cells [73], each containing a pair of $M_{F, k}$ and $M_{C r, k}$ values. These values are used to sample new parameters for $F$ and $C r$ used in mutation and crossover, respectively. The sampling is performed by using the values in a randomly chosen historical cell with index $k \in[1, H]$ in the following way:

$$
\begin{gathered}
F=\operatorname{randc}\left(M_{F, k}, 0.1\right), \\
C r=\operatorname{randn}\left(M_{C r, k}, 0.1\right),
\end{gathered}
$$

where $\operatorname{rand} c(m, s)$ is the Cauchy distributed random value and $\operatorname{randn}(m, s)$ is a normally distributed random value, with position and scale parameters $m$ and $s$ respectively. If the value generated for $\mathrm{Cr}$ is outside $[0,1]$, it is truncated to this range. If the generated value $F>1$, then it is set to 1 , however, in case if $F<0$, it is generated again, until it gets within the range $[0,1]$, as $F=0$ means no mutation.

The successful values of $F$ and $C r$, i.e. those giving an improvement in terms of fitness value, are stored into $S_{F}$ and $S_{C r}$ arrays, as well as the improvement values $\Delta f=|f(u)-f(x)|$. These values are used to update the memory cell with index $h$, which is incremented by 1 every generation, and when $h$ exceeds memory size $H$, it is reset back to 1 . The memory cells for both $F$ and $\mathrm{Cr}$ are updated in a similar way with weighted Lehmer mean:

$$
\text { mean }_{w L}=\frac{\Sigma_{j=1}^{|S|} w_{j} S_{j}^{2}}{\Sigma_{j=1}^{|S|} w_{j} S_{j}},
$$

where $w_{j}=\frac{\Delta f_{j}}{\Sigma_{k=1}^{|S|} \Delta f_{k}}$ and $S$ is either $S_{C r}$ or $S_{F}$. The previous values in the memory cells are replaced by new ones.

In addition to the success-history adaptation for $\mathrm{F}$ and $\mathrm{Cr}$ parameters, the L-SHADE algorithm uses the Linear Population Size Reduction (LPSR) technique. In this method the population size NP is initially set relatively large, and it is recalculated at the end of each generation. The new population size follows the linear function, depending on the available computational resource:

$$
N P_{g+1}=\operatorname{round}\left(\frac{N P_{\text {min }}-N P_{\text {max }}}{N F E_{\text {max }}} N F E+N P_{\text {max }}\right),
$$

where $N P_{\text {min }}=4$ and $N P_{\text {max }}$ are the minimal and initial population sizes, $N F E$ and $N F E_{\text {max }}$ are the current and maximal number of function evaluations. At the end of each generation none, one or several worst individuals are removed from the population.

The standard L-SHADE algorithm also maintains an archive of inferior solutions $A$, which contains parent solutions replaced by better offspring. The archive is initially empty, and it is filled during the selection step: if an offspring is better than its parent, then the parent is stored in the archive. Once the archive reaches its maximum size $N A$, usually equal to the population size $N P$, its update scheme is changed: the parent individuals that 
should be put into the archive replace randomly chosen archive members. The archive size $N A$ is reduced in the same manner as the population size $N P$.

\subsection{Genetic Algorithm for Continuous Optimization}

The Genetic Algorithms were originally proposed for binary or discrete optimization; however, there were multiple attempts to make them work in continuous search spaces. In one of the earliest studies [75], the Simulated Binary Crossover (SBX) was proposed: it imitates the behaviour of the single-point binary crossover operator. Among the existing crossover schemes the SBX has received significant attention and is widely used together with the polynomial mutation strategy [76]. Further on the description of a classical scheme of the GA approach with mentioned search operators will be given.

The continuous genetic algorithm starts with randomly initializing a set of NP solutions within the borders $\left[x \min _{j}, x \max _{j}\right]$. After fitness calculation and remembering the best solution the main loop of the algorithm starts. The main loop contains the following steps:

1. selection of parents for crossover;

2. crossover of parents to generate offspring solutions;

3. mutation of the offspring solutions;

4. replacement of parents with the offspring.

There are several known selection schemes, such as proportional selection, rank-based selection and tournament selection. The last one is used more often due to its simplicity: a set of $T$ individuals is randomly chosen from the population, and the individual having the best fitness value (winner of the tournament) becomes one of the parents. Typically, $T=2$ (binary tournament selection). For each crossover two parents $x^{1}$ and $x^{2}$ are chosen.

The SBX is performed on a pair of parents to produce offspring solutions using the following procedure [77]. First, a uniformly distributed random number $u$ is generated in $[0,1]$, next the $\beta_{q}$ value is calculated as follows:

$$
\beta_{q}=\left\{\begin{array}{ll}
(2 u)^{\frac{1}{n+1}}, & \text { if } u<0.5 \\
\frac{1}{2(1-u)^{\frac{1}{n+1}}}, & \text { otherwise }
\end{array},\right.
$$

where $\eta$ is the non-negative crossover rate parameter that is responsible for the offspring spread, smaller $\eta$ leads to larger spread. The value $\beta_{q}$ is used to calculate two offspring solutions:

$$
\begin{aligned}
& t_{j}^{1}=0.5\left[\left(1+\beta_{q}\right) x_{j}^{1}+\left(1-\beta_{q}\right) x_{j}^{2}\right], \\
& t_{j}^{2}=0.5\left[\left(1-\beta_{q}\right) x_{j}^{1}+\left(1+\beta_{q}\right) x_{j}^{2}\right],
\end{aligned}
$$

where $t_{j}^{1}$ and $t_{j}^{2}$ are the offspring coordinates for variable $j=1,2 \ldots D$. The generated offspring solutions are symmetric about their parents, so no bias towards one of them is present. One of the offspring solutions is randomly chosen to be mutated and moved to the text generation.

Although the SBX generates solutions following a specified probability distribution, i.e., the offspring solutions are sampled around parents, the mutation step could be also used. The polynomial mutation [78] works in a similar way. First a random value $u$ is sampled uniformly within $[0,1]$. Next, the $\delta_{q}$ parameter is calculated:

$$
\delta_{q}= \begin{cases}{\left[2 u+(1-2 u)(1-\delta)^{\left(\eta_{m}+1\right)}\right]^{\frac{1}{n_{m}+1}}-1,} & \text { if } u<0.5, \\ 1-\left[2(1-u)+2(u-0.5)(1-\delta)^{\left(n_{m}+1\right)}\right]^{\frac{1}{n_{m}+1}}, & \text { otherwise }\end{cases}
$$


where $\delta=\frac{\min \left(t_{j}-x \min _{j}, x \max _{j}-t_{j}\right)}{x \max _{j}-x \min _{j}}$ and $\eta_{m}$ is the non-negative distribution parameter (smaller values result in smaller dispersion). The mutated individual's coordinate is calculated as follows:

$$
t_{j}=t_{j}+\delta_{q}\left(\max _{j}-x \min _{j}\right) .
$$

The polynomial mutation is applied to each component of the offspring vector with a probability of $1 / D$. After the mutation, the replacement of parents with the offspring is performed. There are several well-known replacement schemes, for example:

1. offspring only;

2. offspring plus the copy of the best individual;

3. the best of offspring solutions and parents.

In the first case, all the offspring solutions $t$ are copied to the parents $x$ population regardless of their fitness values. This may lead to premature convergence and even loss of genetic diversity as well as losing the best known solution, if it is not stored separately. The second scheme makes sure that the best solution, either from the parents or the offspring, is copied to the new population. The third scheme is more conservative: it sorts the joined set of parents and offspring by fitness and selects the best NP solutions. This ensures that the best solution is always in the population, and if all the offspring solutions were unsuccessful, they will not be copied to the new population.

The described general scheme of GA is not the only one possible, and there are numerous known implementations of this algorithm, however, the given description is sufficient for this study.

\subsection{Proposed Approach}

As long as this study is focused on applying optimization methods to a specific realworld engineering problem, i.e., optimizing resonators topology, several optimization techniques were implemented. The algorithms proposed here are based on both GA and DE, described above, and have several modifications. First of all, there will be two search schemes used: based on differential evolution search operators and based on SBX and polynomial mutation. These two search schemes are based on DE and GA, although they do not fully follow the description given above. The main difference is the replacement mechanism, for which there will be three different scenarios considered, namely:

1. the best of the offspring and parents (replacement type $R T=1$ );

2. random fitness-based replacement (replacement type $R T=2$ );

3. pairwise comparison (DE selection, replacement type $R T=3$ ).

The first and third schemes have been already discussed. The second is performed as follows: after the best solution is saved to the population, for each offspring solution, a random parent index $r 1$ is generated in $[1, N P]$, and if the offspring is better than the $r 1$-th parent, the replacement occurs. This replacement scheme combines the first and the third ones.

In the case if the GA approach is used, the linear population size reduction (LPSR) is applied in the same manner as in L-SHADE algorithm. Both $\eta$ and $\eta_{m}$ parameters are fixed during the algorithm run. GA applies SBX and polynomial mutation to parents, selected by tournament selection with $T=2$. The crossover rate $C R_{g}$, i.e. the ratio of parent vectors components altered by SBX, was set to a constant value. The $C R_{g}$ was used to sample $\mathrm{Cr}$ values as the mean parameter in normal distribution with $\varsigma=0.1$, i.e., the $C r$ sampling was similar to the one used in the L-SHADE algorithm. This algorithm will be further called L-CGA - Continuous Genetic Algorithm with LPSR (algorithm type $A T=1$ ).

In the case if the DE is used, the L-SHADE algorithm with current-to-pbest/1 mutation is applied, however, instead of the crossover operator the SBX from GA is applied for target vector $x_{i}$ and trial vectror $u_{i}, i=1, \ldots, N P$. Next, the polynomial mutation is applied with a probability of $\frac{1}{D}$. The SHA is used to tune only $F$ parameter, without tuning $\eta$ and $\eta_{m}$ for SBX and polynomial mutation. The crossover probability $\mathrm{Cr}$ was sampled using $C R_{g}$ parameter as described above. This algorithm will be further referred to as $\mathrm{L}$ - 
SHADGA: Success-History Adaptive Differential Genetic Algorithm with LPSR (algorithm type $A T=2)$. In our algorithms, we use no archive $(N A=0)$.

As it was mentioned above, the goal of the resonator topology optimization in this study is to perform tuning to the different frequency, while keeping the desired parameters. This is a constrained optimization problem, as it is required that the frequency dependence of losses in reflection should not exceed $-14 \mathrm{~dB}$ in the area between $F_{l}$ and $F_{h}$. Moreover, the number of modes should be the same as defined: not smaller and larger than that of the basic topology. Thus, there should be 3 modes for the first resonator, 6 for the second and 5 for the third resonators respectively.

The constrained optimization problems could be solved with evolutionary algorithms in several ways. One of the most popular methods consists in the implementation of the penalty-based constraint handling techniques: the fitness function contains not only the difference between actual and desired frequency and bandwidth, but also a penalty on the number of modes with frequency dependence of losses exceeding $-14 d B$ in reflection. In this study the dynamic penalties method will be used to calculate fitness values as follows:

$$
\begin{gathered}
\text { Fit }\left(x_{i}\right)=f\left(x_{i}\right)+g^{2} P\left(x_{i}\right), \\
P\left(x_{i}\right)=\frac{p e n\left(x_{i}\right)}{5} P M^{2}, \\
f\left(x_{i}\right)=\left|F_{0}^{a c t}\left(x_{i}\right)-F_{0}^{d e s}\right|+100\left|d F^{a c t}\left(x_{i}\right)-d F^{d e s}\right|, \\
\operatorname{pen}\left(x_{i}\right)=p d b\left(x_{i}\right)\left(1+10\left|n m o d^{a c t}\left(x_{i}\right)-n m o d^{d e s}\right|\right)+50\left|n m o d^{a c t}\left(x_{i}\right)-n m o d^{d e s}\right|, \\
p d b\left(x_{i}\right)= \begin{cases}A F C_{1-1,\left[F_{l}, F_{u}\right]}^{\max }+14, & \text { if } A F C_{1-1,\left[F_{l}, F_{u}\right]}^{\max }>-14, \\
0, & \text { otherwise }\end{cases}
\end{gathered}
$$

where $g$ is the current generation number, $P M$ is the penalty magnitude parameter, $F_{0}^{\text {des }}$, $d F^{d e s}$ and $n m o d^{d e s}$ are the desired values for frequency, bandwidth and number of modes respectively, $F_{0}^{a c t}\left(x_{i}\right), d F^{a c t}\left(x_{i}\right)$ and $n m d^{a c t}\left(x_{i}\right)$ are the actual values for solution $x_{i}$.

Although the scaling of the resonator does not allow simple frequency tuning, it is possible to use the scaled solution as the baseline during initialization. To figure out the required scaling to tune the resonator to the area of desired frequency $F_{0}^{\text {des }}$, a set of measurements has been made for the 3-mode U-shaped resonator. The result of $F_{0}^{a c t}$ measurements for different $y_{h}$ values is shown in Figure 7 .

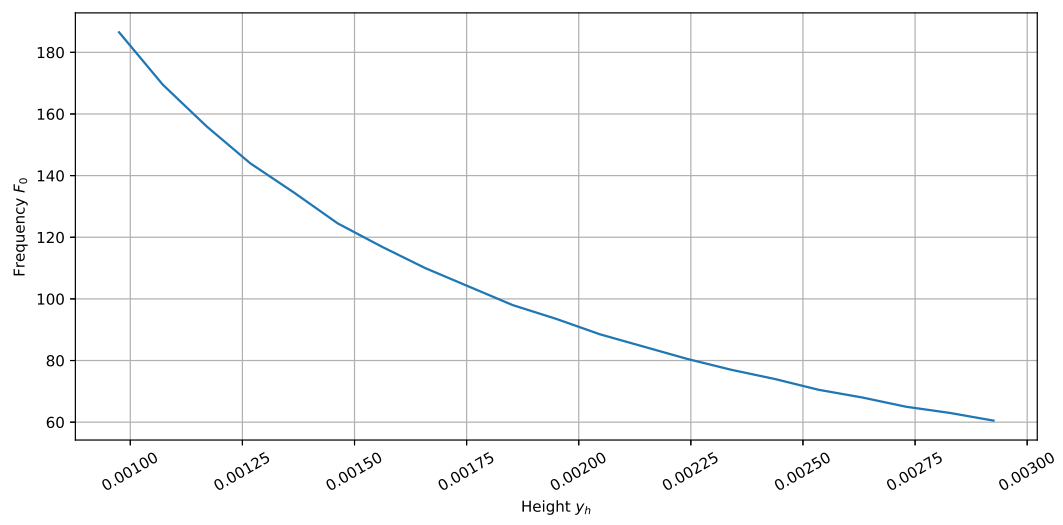

Figure 7. Dependence of $F_{0}^{a c t}$ on $y_{h}$ values.

Based on the observed dependence the following scaling procedure is proposed:

$$
x_{h}^{s c}=x_{h}^{\text {bas }} \frac{F_{0}^{\text {bas }}}{F_{0}^{\text {goal }}},
$$




$$
y_{h}^{s c}=y_{h}^{b a s} \frac{F_{0}^{b a s}}{F_{0}^{g o a l}},
$$

where $x_{h}^{b a s}$ and $y_{h}^{b a s}$ are baseline parameters of the given resonator, $x_{h}^{s c}$ and $y_{h}^{s c}$ are scaled values used during initialization, $F_{0}^{\text {bas }}$ is the frequency of the baseline topology. It should be noted that although in Figure 7 only the dependence on $y_{h}$ is shown, for all three resonators in this study $x_{h}$ does not have such significant influence, however, scaling it as well as $y_{h}$ enables us to find better solutions simpler.

The initialization is performed not uniformly, but close to the scaled solution. In particular, the first individual $x_{1}$ has the scaled $x_{h}$ and $y_{h}$ values, while the rest individuals are generated with normal distribution: $x_{i, j}=x_{1, j} * \operatorname{randn}\left(1,0.02\left(x_{\max }-x \min _{j}\right)\right)$, where $\operatorname{randn}(1, \varsigma)$ is a normally distributed random value with mean equal to 1 and standard deviation $\varsigma$. The parameter value is sampled until it falls within $\left[\operatorname{xmin}_{j}, x \max _{j}\right]$ interval. This initialization allows most of the population to be sampled near the scaled solution, resulting in sufficient diversity, allowing an efficient start of the search process.

In the next section, the details of the performed computational experiments are given, including parameter settings of the used algorithms as well as the final results, approaches comparison, and the applicability of the results for constructing various devices.

\section{Results and Discussion}

One of the most important problems with optimizing the microstrip resonator topology is the computational complexity of the fitness function: modeling the AFC of the resonator in a specified frequency range may take from 5 to $60 \mathrm{~s}$. Resonators used as sensors may have very simply $[15,16,45,46,79]$ or rather sophisticated topology $[17,18,80]$. For more complex topology, the modelling can take more time. Therefore, this should be considered as expensive optimization problem, where every evaluated solution is gained through significant amount of calculations.

The search algorithms were implemented in Matlab and ran on a Windows 10 system with 8-core AMD Ryzen 3700X processor.

One of the problems addressed during the investigation was the development of recommendations about the algorithmic schemes, which would be preferable for resonator topology optimization. In particular, the sensitivity analysis was performed for each of the three baseline resonator topologies. The sensitivity analysis included varying three main parameters for two search algorithms (L-CGA, $A T=1$ and L-SHADGA, $A T=2$ ) with three replacement schemes $(R T=1,2,3)$, including:

1. penalty Magnitude $(P M=1,2,3,4,5)$;

2. crossover and mutation distribution parameters $\left(\eta=\eta_{m}=15,30,45,60,75\right)$;

3. crossover rate parameter $\left(C R_{g}=0.2,0.4,0.6,0.8,1.0\right)$.

Not all the possible combinations were considered due to the significant computational complexity. In particular, for all three baseline resonator topologies, first two combinations of search algorithm $(A T=1,2)$ and three combinations of replacement type $(R T=1,2,3)$ were considered, resulting in a total of six experiments. After this the scheme involving the L-CGA $(A T=1)$ and replacement with the best of offspring and parents $(R T=1)$ was determined as more preferable one. For this scheme the sensitivity analysis was performed, varying the three parameters mentioned above.

The rest of the algorithm parameters were set as follows: initial population size $N P_{\max }=10$, final population size $N P_{\min }=4$, number of function evaluations $N F E_{\max }=500$, historical memory size $H=5$, initial values for memory cells $M F_{k}=0.2$, $k=1, \ldots, H$, tournament size $T=2$. There were 5 independent runs made for every considered case. The optimization problem settings, such as dimensionality $D$ and search ranges, were set depending on the resonator type, shown in Table 1: bas stands for baseline value, and des means desired value that should be reached after optimization. 
Table 1. Algorithm parameters for three cases.

\begin{tabular}{|c|c|c|c|}
\hline Parameter & 3-Mode & 6-Mode & 5-Mode \\
\hline$n m o d^{d e s}$ & 3 & 6 & 5 \\
\hline$D$ & 5 & 7 & 7 \\
\hline$F_{0}^{b a s}$ & 101.5 & 126 & 124.5 \\
\hline$F_{0}^{\text {des }}$ & 150 & 150 & 150 \\
\hline$d F^{b a s}$ & 0.7 & 0.8 & 0.875 \\
\hline$d F^{d e s}$ & 0.7 & 0.8 & 0.875 \\
\hline$x_{h}^{\min }$ & $1 \mathrm{~mm}$ & $1 \mathrm{~mm}$ & $1 \mathrm{~mm}$ \\
\hline$x_{h}^{b a s}$ & $15.5 \mathrm{~mm}$ & $49.5 \mathrm{~mm}$ & $78 \mathrm{~mm}$ \\
\hline$x_{h}^{m a x}$ & $100 \mathrm{~mm}$ & $100 \mathrm{~mm}$ & $100 \mathrm{~mm}$ \\
\hline$y_{h}^{\min }$ & $1 \mathrm{~mm}$ & $1 \mathrm{~mm}$ & $1 \mathrm{~mm}$ \\
\hline$y_{h}^{b a s}$ & $280 \mathrm{~mm}$ & $280 \mathrm{~mm}$ & $93.5 \mathrm{~mm}$ \\
\hline$y_{h}^{\max }$ & $1000 \mathrm{~mm}$ & $1000 \mathrm{~mm}$ & $1000 \mathrm{~mm}$ \\
\hline$x_{s 1}^{\min }$ & 0 & 0 & 0 \\
\hline$x_{s 1}^{b a s}$ & 0.53 & 0.121 & 0.0384 \\
\hline$x_{s 1}^{\max }$ & 1 & 1 & 1 \\
\hline$x_{s 2}^{\min }$ & - & 0 & 0 \\
\hline$x_{s 2}^{b a s}$ & - & 0.969 & 0.075 \\
\hline$x_{s 2}^{\text {max }}$ & - & 1 & 0.5 \\
\hline$y_{s 1}^{\min }$ & 0 & 0 & 0 \\
\hline$y_{s 1}^{b a s}$ & 0.32 & 0.236 & 0.77 \\
\hline$y_{s 1}^{\max }$ & 1 & 1 & 1 \\
\hline$y_{s 2}^{\min }$ & - & 0 & 0.01 \\
\hline$y_{s 2}^{b a s}$ & - & 0.204 & 1.98 \\
\hline$y_{s 2}^{\max }$ & - & 1 & 3 \\
\hline$y_{p}^{\min }$ & 0 & 0 & 0 \\
\hline$y_{p}^{b a s}$ & 0.02 & 0.02 & 0.145 \\
\hline$y_{p}^{\max }$ & 1 & 1 & 1 \\
\hline
\end{tabular}

Tables $2-4$ contain the mean and standard deviation of best achieved values after 5 runs for the first, the second and the third case, i.e., 3-mode U-shaped resonator, 7-mode $\mathrm{U}$-shaped resonator with additional cut and 5-mode $\mathrm{W}$-shaped resonator. The experiments with different algorithm types and replacement types were made with $\eta=45, P M=3$ and $C R_{g}=0.6$. For cases when one of these parameters was varied, the other two were set to these values.

Table 2. Experimental results for 3-mode micrsostrip resonator.

\begin{tabular}{cc}
\hline Parameters & Mean Fitness and Standard Deviation \\
\hline L-CGA, $R T=1$ & $0.67 \pm 0.00$ \\
L-SHADGA, $R T=1$ & $0.67 \pm 0.00$ \\
L-CGA, $R T=2$ & $0.96 \pm 0.59$ \\
L-SHADGA, $R T=2$ & $0.67 \pm 0.00$ \\
L-CGA, $R T=3$ & $10.86 \pm 1.55$ \\
L-SHADGA, $R T=3$ & $5.73 \pm 3.09$ \\
\hline
\end{tabular}


Table 2. Cont.

\begin{tabular}{cc}
\hline Parameters & Mean Fitness and Standard Deviation \\
\hline$P M=1$ & $0.67 \pm 0.00$ \\
$P M=2$ & $0.95 \pm 0.56$ \\
$P M=3$ & $0.67 \pm 0.00$ \\
$P M=4$ & $1.40 \pm 1.22$ \\
$P M=5$ & $0.93 \pm 0.53$ \\
\hline$\eta=15$ & $2.84 \pm 1.85$ \\
$\eta=30$ & $1.09 \pm 0.62$ \\
$\eta=45$ & $0.67 \pm 0.00$ \\
$\eta=60$ & $0.67 \pm 0.00$ \\
$\eta=75$ & $0.67 \pm 0.00$ \\
\hline$C R_{g}=0.2$ & $0.68 \pm 0.03$ \\
$C R_{g}=0.4$ & $1.08 \pm 0.60$ \\
$C R_{g}=0.6$ & $0.67 \pm 0.00$ \\
$C R_{g}=0.8$ & $0.67 \pm 0.00$ \\
$C R_{g}=1.0$ & $0.67 \pm 0.00$ \\
\hline
\end{tabular}

For the case of 3-mode resonator, the best results were achieved with both L-CGA and L-SHADGA with the first replacement scheme, i.e. the best of parents and the offspring. The worst case is the usage of L-CGA with the pairwise comparison replacement scheme. The penalty magnitude does not show any significant influence on the performance, however, the mutation and crossover distribution parameters do, in particular, smaller $\eta$ values result in larger spread of sampled values and worse results. As for the crossover rate, it does not have any significant influence as well.

Table 3. Experimental results for 6-mode micrsostrip resonator.

\begin{tabular}{cc}
\hline Parameters & Mean Fitness and Standard Deviation \\
\hline L-CGA, $R T=1$ & $0.87 \pm 0.52$ \\
L-SHADGA, $R T=1$ & $0.69 \pm 0.62$ \\
L-CGA, $R T=2$ & $0.90 \pm 0.00$ \\
L-SHADGA, $R T=2$ & $1.34 \pm 0.36$ \\
L-CGA, $R T=3$ & $1.38 \pm 0.39$ \\
L-SHADGA, $R T=3$ & $1.49 \pm 0.29$ \\
\hline$P M=1$ & $0.90 \pm 0.00$ \\
$P M=2$ & $1.01 \pm 0.60$ \\
$P M=3$ & $0.87 \pm 0.52$ \\
$P M=4$ & $0.69 \pm 0.62$ \\
$P M=5$ & $1.34 \pm 0.36$ \\
\hline$\eta=15$ & $1.05 \pm 0.29$ \\
$\eta=30$ & $1.32 \pm 0.35$ \\
$\eta=45$ & $0.87 \pm 0.52$ \\
$\eta=60$ & $0.69 \pm 0.62$ \\
$\eta=75$ & $0.87 \pm 0.52$ \\
\hline$C R_{g}=0.2$ & $0.72 \pm 0.36$ \\
$C R_{g}=0.4$ & $1.01 \pm 0.60$ \\
$C R_{g}=0.6$ & $0.87 \pm 0.52$ \\
$C R_{g}=0.8$ & $0.72 \pm 0.36$ \\
$C R_{g}=1.0$ & $0.69 \pm 0.62$ \\
\hline & \\
\hline
\end{tabular}

For the case of 6-mode resonator, the best results were achieved with L-CGA and with the second replacement scheme, i.e. random replacement with comparison. The worst case is the usage of L-SHADGA with the pairwise comparison replacement scheme. Again, the penalty magnitude does not influence the results very much. Larger $\eta$ values usually result in better fitness values and the crossover rate does not have any significant influence. 
Table 4. Experimental results for 5-mode micrsostrip resonator.

\begin{tabular}{cc}
\hline Parameters & Mean Fitness and Standard Deviation \\
\hline L-CGA, $R T=1$ & $1.34 \pm 0.77$ \\
L-SHADGA, $R T=1$ & $1.44 \pm 0.39$ \\
L-CGA, $R T=2$ & $1.39 \pm 0.28$ \\
L-SHADGA, $R T=2$ & $1.39 \pm 0.37$ \\
L-CGA, $R T=3$ & $4.42 \pm 2.10$ \\
L-SHADGA, $R T=3$ & $1.80 \pm 0.58$ \\
\hline$P M=1$ & $1.34 \pm 0.52$ \\
$P M=2$ & $0.89 \pm 0.31$ \\
$P M=3$ & $1.34 \pm 0.77$ \\
$P M=4$ & $1.77 \pm 0.77$ \\
$P M=5$ & $1.10 \pm 0.30$ \\
\hline$\eta=15$ & $1.68 \pm 0.54$ \\
$\eta=30$ & $1.19 \pm 0.53$ \\
$\eta=45$ & $1.34 \pm 0.77$ \\
$\eta=60$ & $1.02 \pm 0.66$ \\
$\eta=75$ & $1.24 \pm 0.52$ \\
\hline$C R_{g}=0.2$ & $0.74 \pm 0.28$ \\
$C R_{g}=0.4$ & $1.36 \pm 1.32$ \\
$C R_{g}=0.6$ & $1.34 \pm 0.77$ \\
$C R_{g}=0.8$ & $1.40 \pm 0.70$ \\
$C R_{g}=1.0$ & $1.32 \pm 0.59$
\end{tabular}

For the case of 5-mode resonator, the best results were achieved with L-CGA and with the first replacement scheme, the best of parents and offspring. The worst case is again the usage of L-CGA with the pairwise comparison. The sensitivity analysis for three other parameters has similar results: larger $\eta$ values are better, penalty magnitude does not show any significant influence, however, smaller $C R_{g}$ values appear to be better.

The fitness values, which are close to 1 or even smaller, are achieved usually due to small inaccuracy in $F_{0}$ frequency tuning, because the smallest possible step for $F_{0}$ is 0.5 . In all cases the considered algorithms were able to find solutions that satisfied the constraints. Figures 1 and 2 show the tuned topology of the 3-mode resonator, where all the parameters were found with the L-CGA algorithm using first replacement scheme.

Figures 8-10 contain the convergence graphs for all the performed experiments.
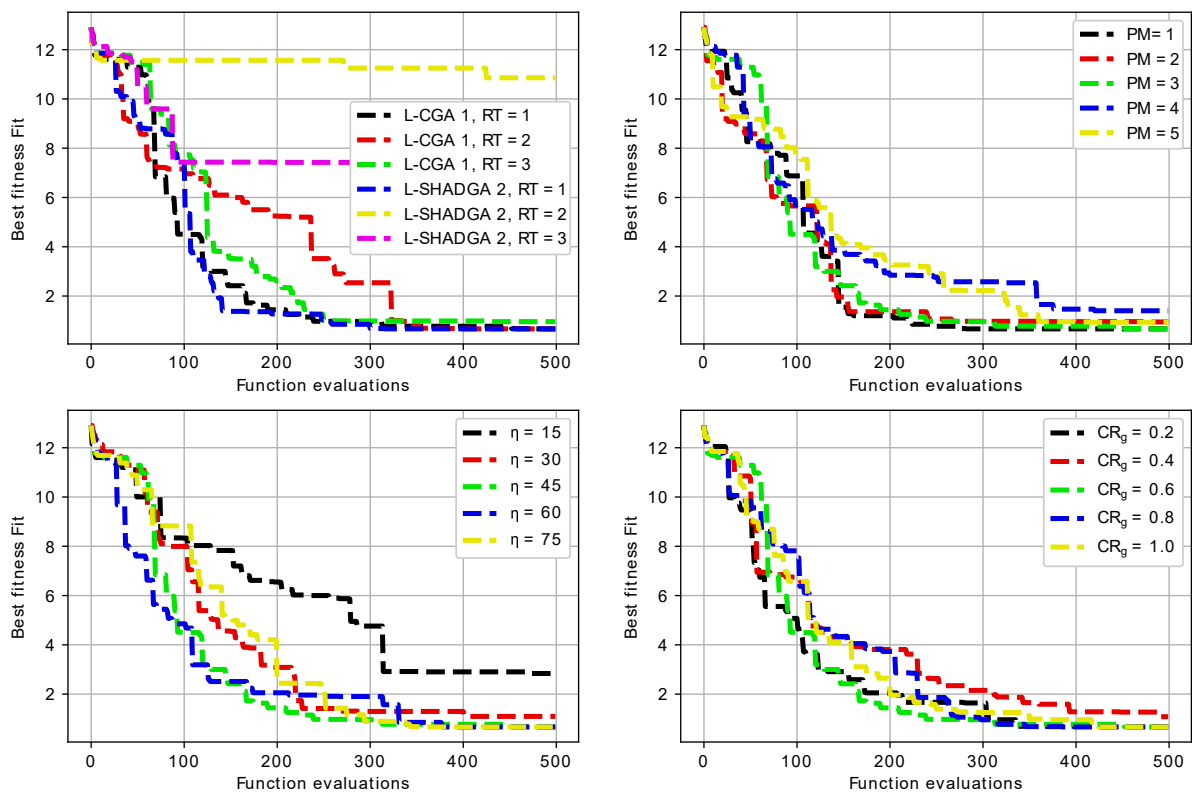

Figure 8. Convergence graphs of algorithms with different parameters for 3-mode resonator. 

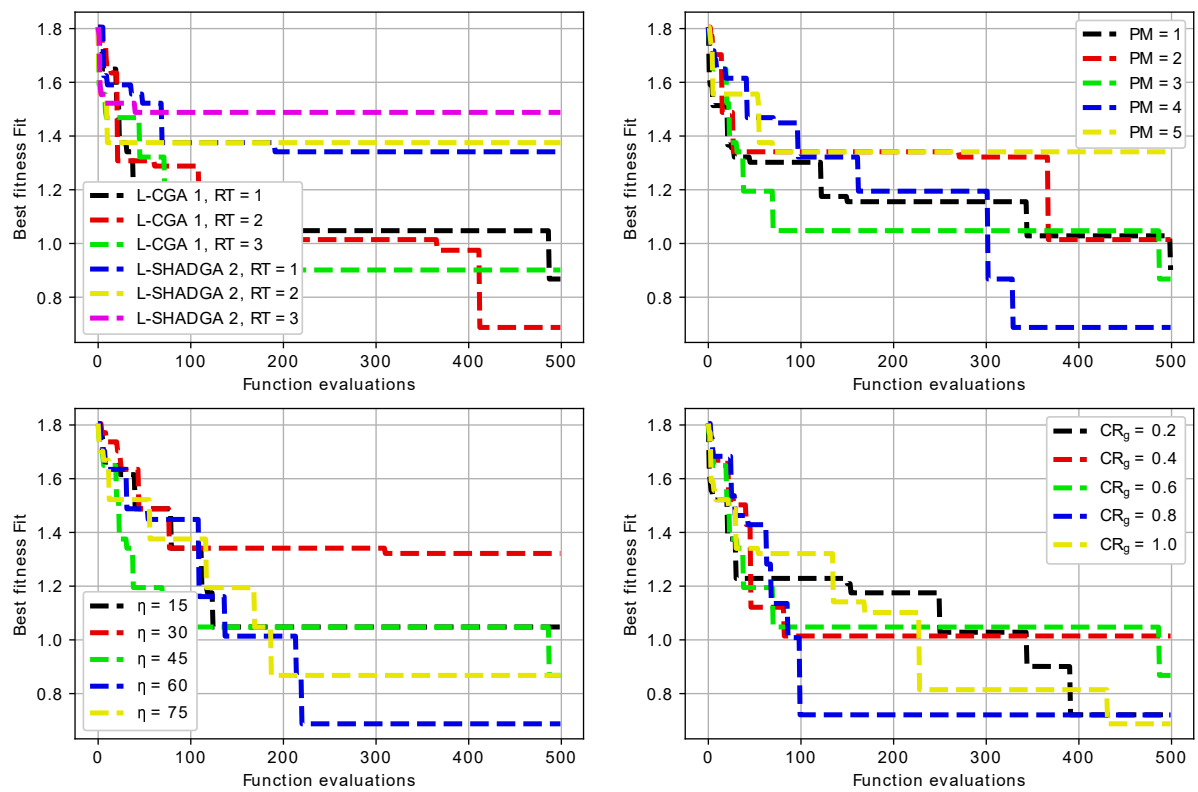

Figure 9. Convergence graphs of algorithms with different parameters for 6-mode resonator.
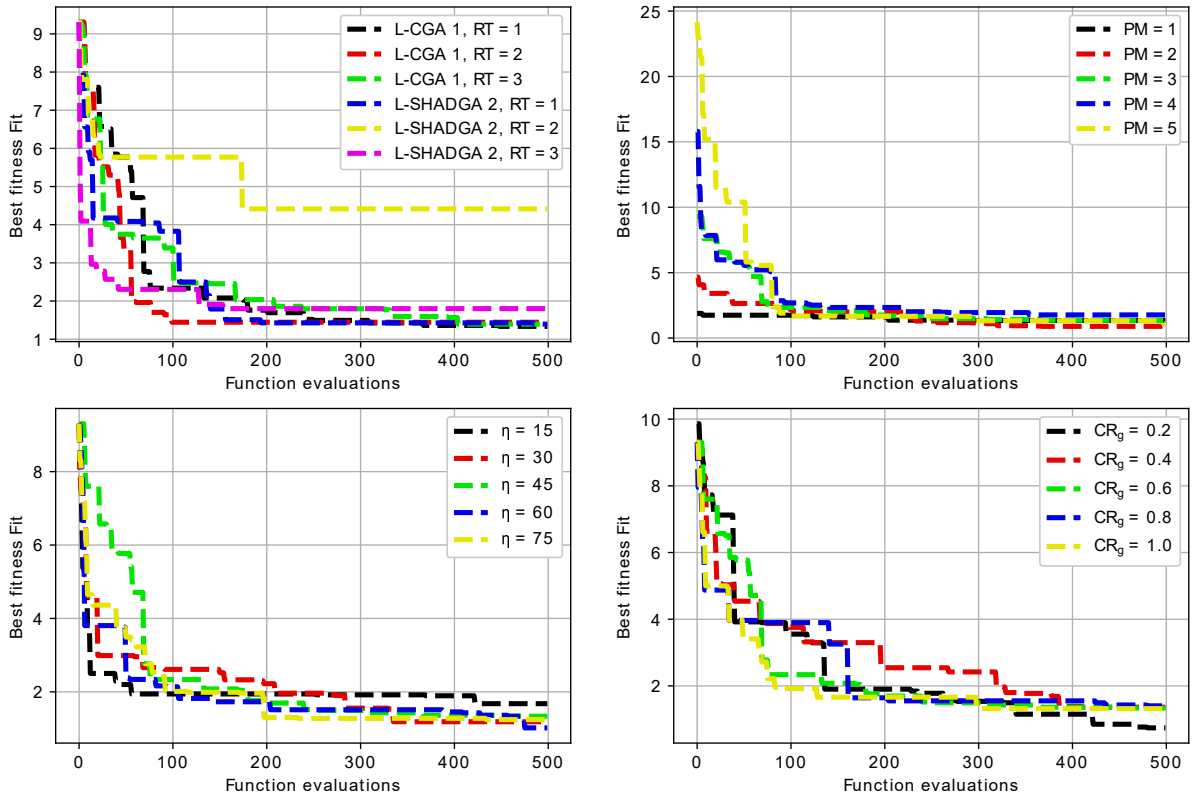

Figure 10. Convergence graphs of algorithms with different parameters for 5-mode resonator.

Figures 8-10 show that all the considered algorithms were able to converge after around 300 generations in most cases. For the 3-mode resonator case, the difference between baseline and the desired frequency was larger, so the search for the optimal topology took longer. For the 6-mode and 5-mode cases the difference was not so large, and the solutions were found earlier.

In addition to the performed experiments, the optimization methods available in Matlab optimization toolbox were considered. Due to the fact that the considered problem is not smooth in terms of fitness values and cannot be differentiated, some of the methods were not able to solve such problem. The only method that was able to show competitive results was the Simulated Annealing (SA) algorithm, which was tested for all three cases with a computational resource of 500 function evaluations and recommended parameters. The same scaling of resonator size was applied to initialize the algorithm. Table 5 contains the fitness values achieved by the SA algorithm. 
Table 5. Experimental results of the Simulated Annealing algorithm.

\begin{tabular}{cc}
\hline Problem & Mean Fitness and Standard Deviation \\
\hline 3-mode resonator & $3.33 \pm 1.46$ \\
6-mode resonator & $0.87 \pm 0.52$ \\
5-mode resonator & $2.91 \pm 0.65$ \\
\hline
\end{tabular}

The SA algorithm was able to solve only the second problem, which appeared to be relatively simple, considering the convergence graphs in Figure 9. The best solutions for the first problem are significantly worse, and have much larger standard deviation, probably due to the large difference between baseline and the desired bandwidth. Comparing one of the best variants of evolutionary algorithms, L-CGA with the first replacement scheme, it should be noted that the fitness level achieved by simulated annealing (3.33) is reached after around 120 function evaluations, i.e., $25 \%$ of the total computational resource for the 3-mode resonator case. For the 6-mode case the results are comparable and reached almost with the same resource, but for 5-mode resonator case the L-CGA was able to get to the same fitness values level at around 75 generations, or $15 \%$ of the total computational resource.

For the practical use of our approach, it is much more important for the algorithm to achieve the desired values of the criteria $\left(F_{0}\right.$ and $\left.d F\right)$. For other types of microwave devices which can be used as sensors, in radars, antennas and sensor network nodes, these criteria may differ. In our research, we consider $F_{0}$ and $d F$ as examples of possible criteria. Our approach requires these criteria to be functionals of the AFC. Nevertheless, the central frequency and the width of the passband are essential for the majority of sensors and resonators regardless of their purpose. For example, for the gas sensors, the central frequency $F_{0}$ is determined by the properties of the gas to be detected. For a deeper comparison of approaches, the achieved frequency parameters are considered. In Tables 6-8 the best $F_{0}^{a c t}$ and $d F^{a c t}$ of both L-CGA with first replacement scheme and SA algorithm are given for all program runs.

Table 6. Comparison of resonator characteristics achieved by L-CGA and SA, 3 mode case.

\begin{tabular}{ccccc}
\hline \multirow{2}{*}{ Run } & \multicolumn{2}{c}{ L-CGA } & \multicolumn{2}{c}{ SA } \\
\cline { 2 - 5 } & $\boldsymbol{F}_{\mathbf{0}}^{\text {act }}$ & $\boldsymbol{d F ^ { a c t }}$ & $\boldsymbol{F}_{\mathbf{0}}^{\text {act }}$ & $\boldsymbol{d F}^{\text {act }}$ \\
\hline Run 1 & 150 & 0.70667 & 150 & 0.69333 \\
Run 2 & 150 & 0.69333 & 150 & 0.66667 \\
Run 3 & 150 & 0.69333 & 150 & 0.65333 \\
Run 4 & 150 & 0.69333 & 150 & 0.65333 \\
Run 5 & 150 & 0.69333 & 150 & 0.66667 \\
\hline Mean & 150 & 0.696 & 150 & 0.6667 \\
Std & 0 & 0.005 & 0 & 0.015 \\
\hline
\end{tabular}

For the 3-mode resonator case, both algorithms were able to get the desired $F_{0}^{a c t}$, however the L-CGA obtained much better $d F_{0}^{a c t}$ values, i.e., closer to the desired 0.7. Moreover, the standard deviation of $d F_{0}^{a c t}$ values was smaller. 
Table 7. Comparison of resonator characteristics achieved by L-CGA and SA, 6 mode case.

\begin{tabular}{ccccc}
\hline \multirow{2}{*}{ Run } & \multicolumn{2}{c}{ L-CGA } & \multicolumn{2}{c}{ SA } \\
\cline { 2 - 5 } & $\boldsymbol{F}_{\mathbf{0}}^{\text {act }}$ & $\boldsymbol{d} \boldsymbol{F}^{\text {act }}$ & $\boldsymbol{F}_{\mathbf{0}}^{\text {act }}$ & $\boldsymbol{d F}^{\text {act }}$ \\
\hline Run 1 & 149.5 & 0.79599 & 150.5 & 0.80399 \\
Run 2 & 148.5 & 0.80135 & 150.5 & 0.80399 \\
Run 3 & 150 & 0.8 & 150 & 0.8 \\
Run 4 & 149.5 & 0.79599 & 148.5 & 0.80135 \\
Run 5 & 149.5 & 0.79599 & 150.5 & 0.80399 \\
\hline Mean & 149.4 & 0.7978 & 150 & 0.8026 \\
Std & 0.490 & 0.002 & 0.774 & 0.0017 \\
\hline
\end{tabular}

In the 6-mode case, the average of the results received by SA is exactly 150 , however the standard deviation is larger, while L-CGA got more stable results. As for the $d F_{0}^{a c t}$ values, the mean and standard deviations in this case are similar, which explains similar performance in this case.

Table 8. Comparison of resonator characteristics achieved by L-CGA and SA, 5 mode case.

\begin{tabular}{ccccc}
\hline \multirow{2}{*}{ Run } & \multicolumn{2}{c}{ L-CGA } & \multicolumn{2}{c}{ SA } \\
\cline { 2 - 5 } & $\boldsymbol{F}_{\mathbf{0}}^{\text {act }}$ & $\boldsymbol{d} \boldsymbol{F}^{\text {act }}$ & $\boldsymbol{F}_{\mathbf{0}}^{\text {act }}$ & $\boldsymbol{d F}^{\text {act }}$ \\
\hline Run 1 & 149 & 0.88591 & 151 & 0.87417 \\
Run 2 & 150 & 0.88 & 152 & 0.86842 \\
Run 3 & 150 & 0.88 & 150 & 0.86667 \\
Run 4 & 148 & 0.87838 & 149 & 0.87248 \\
Run 5 & 149 & 0.87248 & 151 & 0.87417 \\
\hline Mean & 149.2 & 0.8793 & 150.6 & 0.8712 \\
Std & 0.748 & 0.004 & 1.019 & 0.003 \\
\hline
\end{tabular}

For the case of 5-mode W-shaped resonator, the average $F_{0}^{a c t}$ of L-CGA is not as close to the desired 150 as that of the SA, however, the standard deviation is again smaller. At the same time the $d F_{0}^{a c t}$ achieved by L-CGA are closer to the desired, and the standard deviations are comparable. In general, the L-CGA appears to show more stable results and is able to get to the optimal values or very close to them for both frequency and delta.

Considering these results, it can be concluded, that the developed approaches could be used for multimode resonator topology optimization when tuning a known structure to the new frequency range. The approaches presented in this study are general, and could be used for any other type of structure optimization, i.e., other resonators, filters, sensors, radars, antennas etc.

Although the proposed algorithms have shown to perform better than some classical approaches, it is important to consider and discuss the complexity of the optimization problems involving resonator topology optimization. To visualize the landscape of the main parameters, an additional experiment with the 5-mode resonator was performed: the two main parameters responsible for the size, namely $x_{h}$ and $y_{h}$ were varies within $5 \%$ margin from the scaled solution, i.e., the one used during initialization. Figure 11 shows the dependence of $F_{0}^{a c t}$ on the resonator size. 

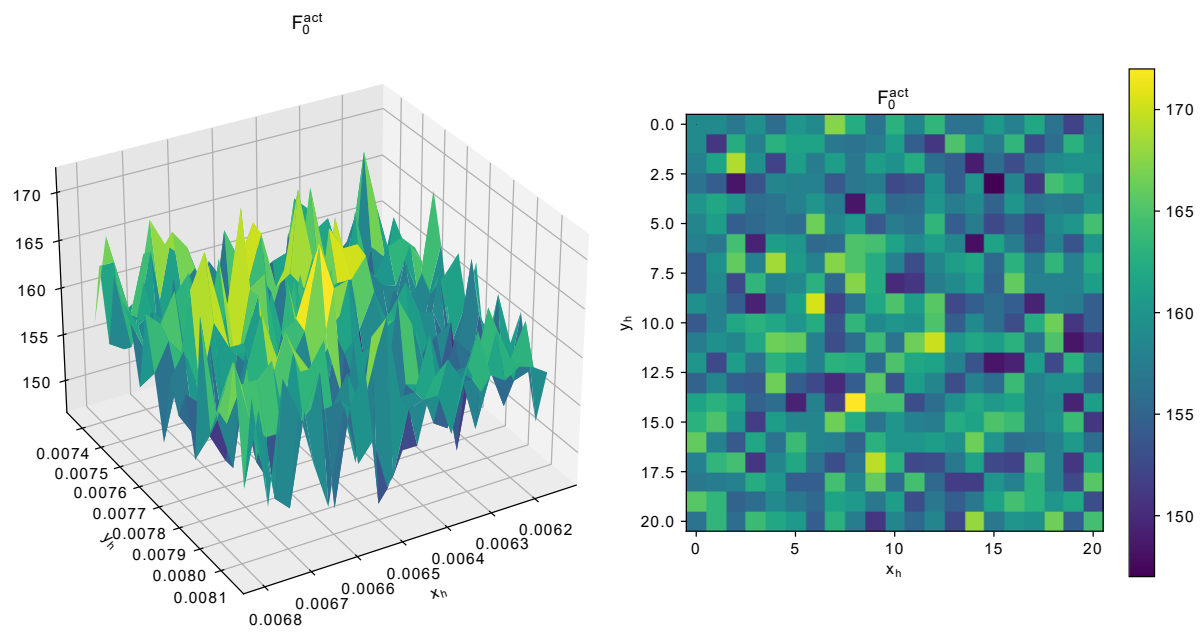

Figure 11. Dependence of $F_{0}^{a c t}$ around scaled solution for 5-mode resonator.

As can be seen from Figure 11, the landscape of the function is rather rough, that is there are many local optima, and even a small change in geometrical characteristics may cause significant change in $F_{0}$, which jumps from 150 to 170 . Figures 12 and 13 show similar graphs for $d F$ and number of modes.
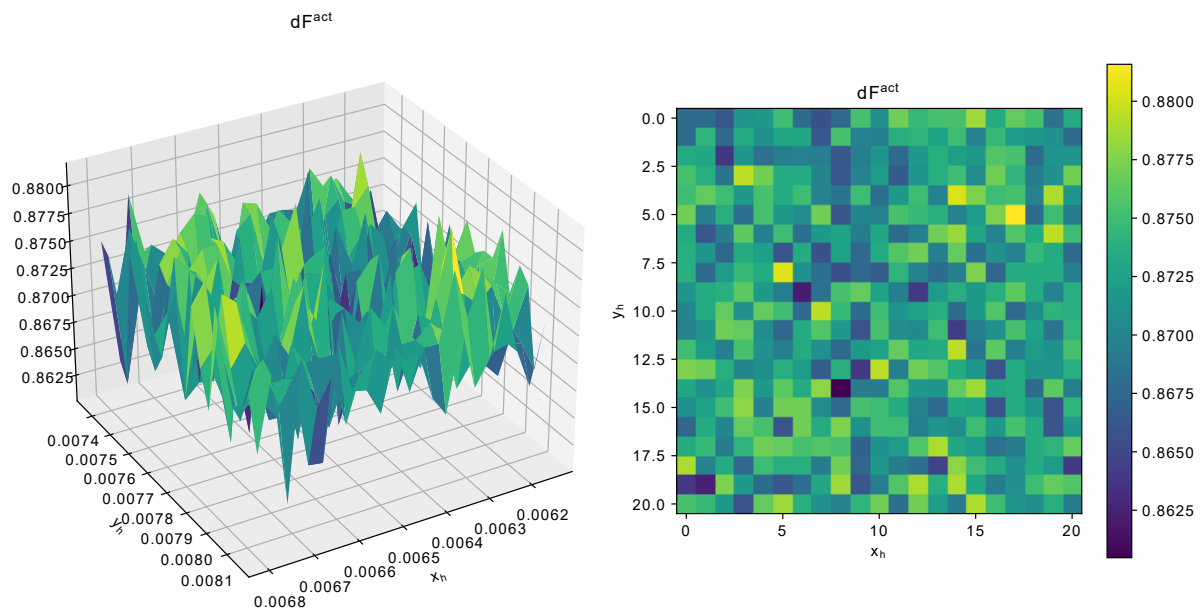

Figure 12. Dependence of $d F^{a c t}$ around scaled solution for 5-mode resonator.

nmod
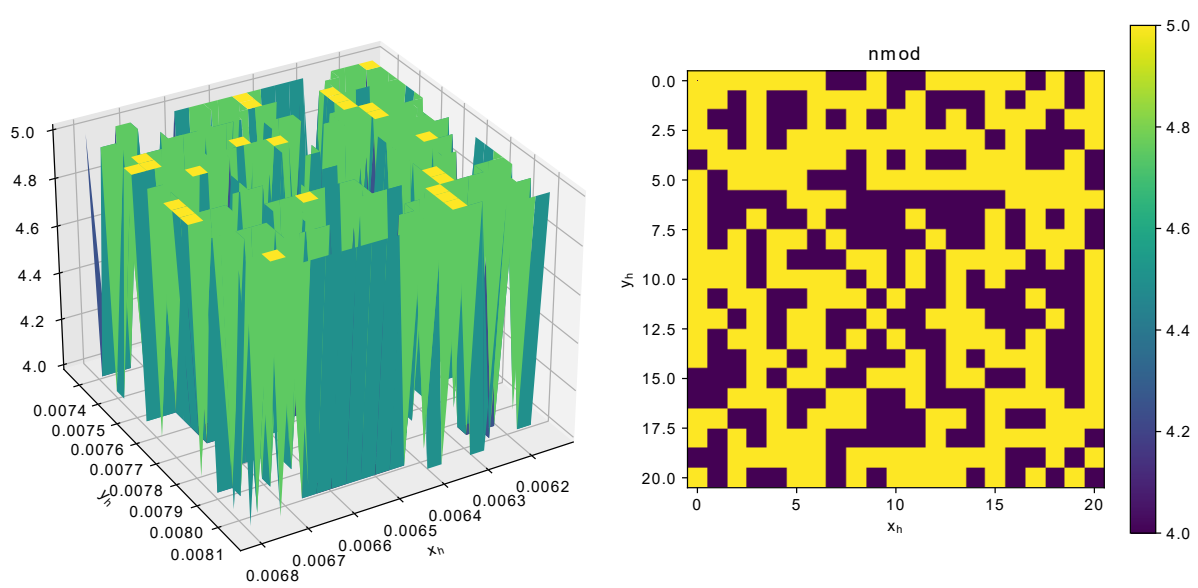

Figure 13. Dependence of number of modes around scaled solution for 5-mode resonator. 
Considering the landscape in Figures 12 and 13, the value of $d F$ again has multiple local optima, but the difference between worst and best parameters is not so signficiant. As for the number of modes, changing either $x_{h}$ or $y_{h}$ may cause the resonator switch to 4 mode, and it may be quite difficult to get 5 modes. Figure 14 demonstrates the $A F C_{1-1}^{\max }$ values, resposible for penalizing the solutions.
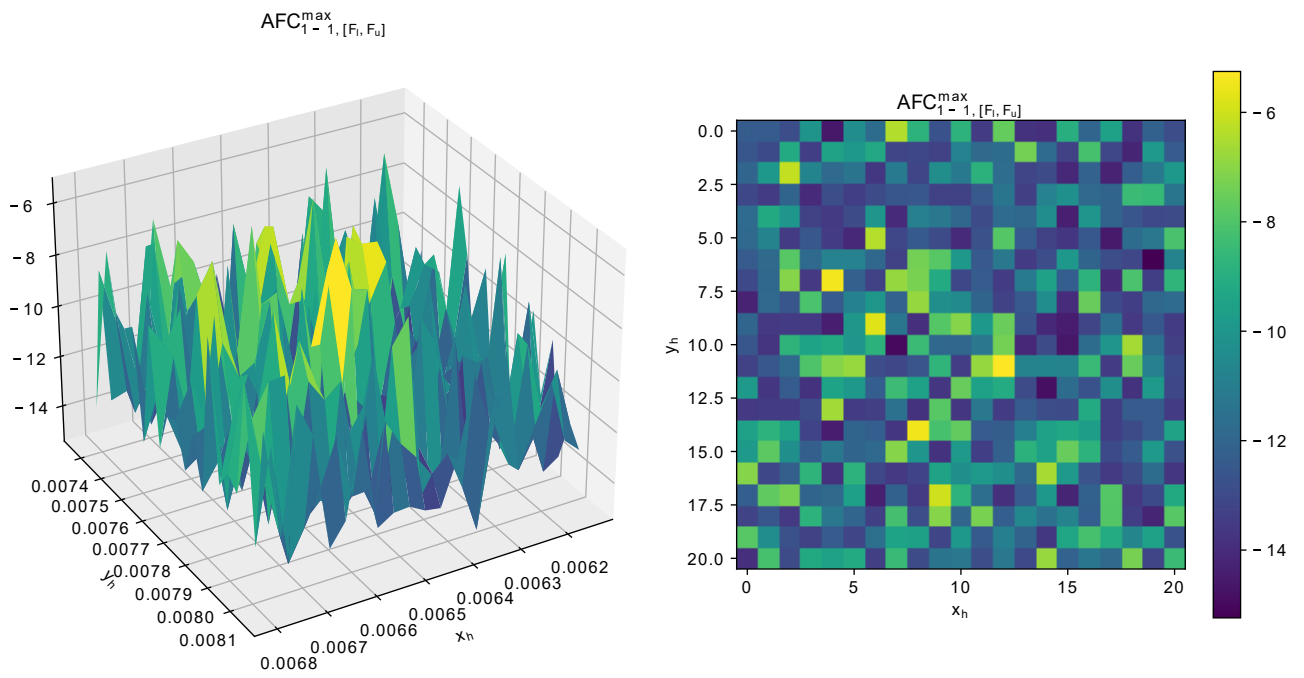

Figure 14. Dependence of $A F C_{1-1,\left[F_{l}, F_{u}\right]}^{\max }$ around scaled solution for 5-mode resonator.

The landscape of measured $A F C_{1-1,\left[F_{l}, F_{u}\right]}^{\max }$ with different $x_{h}$ or $y_{h}$ around the scaled solution shows that this constraint, i.e., it should be less then -14 , could be difficult to satisfy, as some of the points may get up to -6 .

The analysis of landscape near the scaled solutions shows that searching for the appropriate configuration of parameter values may be difficult, as even small changes may result in very different solutions. Here, only $x_{h}$ or $y_{h}$ were considered, and the landscape analysis of other parameters, such as $x_{s 1}$ or $y_{s 1}$ could give some additional insights. However, considering the dimensionality and the computational complexity of the problem, it can be concluded that applying developed algorithms justified.

Our research is focused on optimization of flat resonator constructions. However, the described approach does not require the resonator structure to be planar only. The only difference is that the process of electrodynamic modelling of the $3 \mathrm{D}$ resonators is much more time consuming which limits the usage of our approach because of the need to run such a simulation many times. Nevertheless, the rapid growth of the computing facilities gives us a reasonable hope to use similar approach to a wider variety of shapes including 3D ones in future. The progress in the development of the 3D printing technology enables us to fabricate special microwave devices including sensors and their parts [81-83] for specific needs, according to individual orders. The use of biocompatible materials makes it possible to use such 3D printed devices [84] as immunosensors [85], toxicity [86] and other types of sensors. These types of sensors include both 3D spatial designs and planar designs. Our approach can be directly implemented to the planar ones.

The proposed approach to the automatic design of microwave devices includes the following stages:

Stage 1: Definition of the criteria and their desired values, and the constraints (both the criteria and left parts of the constraints must be functionals of the AFC);

Stage 2: Drawing a perspective device topology with arbitrary geometric dimensions in a specialized software tool for electrodynamic modeling;

Stage 3: Defining the variable geometric parameters to be adjusted and feasible ranges of their values;

Stage 4: Running the optimization algorithm; 
Stage 5: investigation and validation of the AFC and achieved values of the criteria, return to Stage 2 if the desired values of the criteria are not achieved or the solution is infeasible;

Stage 6: Manufacturing and testing a prototype of the device.

The filters and other microwave devices used in radar sensors and sensor network nodes may be composed of several resonators [24,46]. The properties of the device depend on the topology of the resonators, number of resonators and their interconnections. Thus, for such complex cases, the optimization problem must contain both real and discrete variables. Since the DE algorithms are aimed at optimization of continuous functions, such problems may require more complex algorithms to be developed and implemented in future research. Nevertheless, the parameter adjustment of the existing topologies for the desired frequency and bandpass is an important problem, and our study greatly facilitates the solution of this problem.

The proposed algorithmic approach involves electrodynamic analysis systems of microwave device structures which is capable of simulating the amplitude-frequency response with high accuracy $[12,87]$. For the planar microstrip filters, electrodynamic analysis shows a good agreement of the measured and calculated characteristics of the devices (the error in the response does not exceed 3-6\% (see Figures 2, 4 and 6) which allows us to use the results of the proposed algorithm as an accurate estimation of the characteristics of the frequency-selective properties for a device to be manufactured.

\section{Conclusions}

In this study, an important engineering problem of optimizing the topology of microstrip microwave devices was considered. To be able to solve this problem, characterized by a complex and computationally expensive fitness function and a set of non-linear constraints, we proposed two algorithms with a set of variations in algorithmic schemes and parameter values. The performed experimental comparison of the proposed algorithms on three cases, including the 3-mode U-shaped resonator, 6-mode U-shaped resonator with additional cut and 5-mode W-shaped resonator, have shown that the proposed algorithms are capable of finding optimal solutions for these topologies with relatively low computational resource. We proposed an algorithmic approach which enables us to perform automatic design of the microstrip resonators in accordance with the desired values of the critera which must be formulated as functionals of the AFC. This approach involves the electrodynamic modelling tools for estimating the AFC based on the values of the variable dimensions of the resonator topology.

We implemented our approach to three types of resonators with rather simple shapes which are nevertheless widely used in sensors, filters and radar antennas. The considered resonator types have wide bandwidth and, therefore, are promising for building selective devices with extended bandwidths, as well as sensors with an extended frequency range. However, our approach is in no way tied to the specifics of the structures we used as examples, and may be implemented for adjusting parameters of other resonator shapes as well as investigating the features of new perspective topologies

We used the center frequency $F_{0}$ and the width of the passband $d F$ as the examples of possible criteria which may be set for the devices. Our criteria are commonly used (to obtain a narrow bandwidth commonly used in specific sensors, we must set a very low value of $d F$ ). For investigation of other perspective topologies of the resonators used as microwave sensors, parts of radar antennas or filters, new criteria may be formulated as functionals of the the AFC.

Further developments in this area may also include (but are not limited to) experimenting with self-adaptive genetic search operators, including improved local search mechanisms as well as applying surrogate models to approximate the fitness function landscape. 
Author Contributions: Conceptualization, V.V.S., S.A.K., L.A.K. and A.M.P.; methodology, S.A.K., V.V.S. and L.A.K.; software, V.V.S. and L.A.K.; validation, S.A.K., V.V.S. and L.A.K.; formal analysis, V.V.S., S.A.K. and A.M.P.; investigation, V.V.S. and L.A.K.; resources, V.V.S.; data curation, V.V.S.; writing-original draft preparation, V.V.S. and L.A.K.; writing-review and editing, L.A.K., V.V.S. and S.A.K.; visualization, V.V.S., S.A.K., L.A.K. and A.M.P.; supervision, L.A.K.; project administration, L.A.K.; funding acquisition, A.M.P. All authors have read and agreed to the published version of the manuscript.

Funding: This work was funded by the Ministry of Science and Higher Education of the Russian Federation, State Contract FEFE-2020-0013.

Institutional Review Board Statement: Not applicable.

Data Availability Statement: Not applicable.

Conflicts of Interest: The authors declare no conflict of interest.

\section{Abbreviations}

The following abbreviations are used in this manuscript:

$\begin{array}{ll}\text { AFC } & \text { amplitude-frequency characteristic } \\ \text { HPF } & \text { high-pass filter } \\ \text { EA } & \text { Evolutionary Algorithm } \\ \text { GA } & \text { Genetic Algorithm } \\ \text { PSO } & \text { Particle Swarm Optimization } \\ \text { BCH } & \text { bound constraint handling } \\ \text { SHA } & \text { success-history adaptation } \\ \text { LPSR } & \text { linear population size reduction } \\ \text { SBX } & \text { simulated binary crossover }\end{array}$

\section{References}

1. Belyaev, B.A.; Khodenkov, S.A.; Galeev, R.G.; Shabanov, V.F. Investigation of microstrip structures of wideband bandpass filters. Dokl. Phys. 2015, 60, 95-101. [CrossRef]

2. Praveen, K.R.; Rajan, M. Resonator Based Antenna Sensor for Breast Cancer Detection. Prog. Electromagn. Res. M 2021, 101, 149-159. [CrossRef]

3. Tang, J.; Zhu, Q.; Li, C.; Chen, Y.; Zhang, Z.; Guo, H.; Nian, F.; Liu, W.; Cai, A.; Yang, B.; Liu, J. A U-shaped microwave resonator for flexible mechanical sensors application. Rev. Sci. Instrum. 2019, 90, 114712. [CrossRef]

4. $\quad$ Delwar, T.S.; Siddique, A.; Biswal, M.R.; Behera, P.; Choi, Y.; Ryu, J.Y. A 65 nm Duplex Transconductance Path Up-Conversion Mixer for $24 \mathrm{GHz}$ Automotive Short-Range Radar Sensor Applications. Sensors 2022, 22, 594. [CrossRef] [PubMed]

5. Hong, J.S.; Lancaster, M.J. Design of highly selective microstrip bandpass filters with a single pair of attenuation poles at finite frequencies. IEEE Trans. Microw. Theory Tech. 2000, 48, 1089-1107.

6. Belyaev, B.A.; Khodenkov, S.A.; Shabanov, V.F. Investigation of frequency-selective devices based on a microstrip 2D photonic crystal. Dokl. Phys. 2016, 61, 155-159. [CrossRef]

7. Ohira, M.; Hashimoto, S.; Ma, Z.; Wang, X. Coupling-matrixbased systematic design of single-DC-bias-controlled microstrip higher order tunable bandpass filters with constant absolute bandwidth and transmission zeros. IEEE Trans. Microw. Theory Tech. 2019, 67, 118-128. [CrossRef]

8. Chen, Z.; Li, K.; Qu, D.; Zhong, X.; Sun, L.; Ma, W. A novel microstrip absorptive bandstop filter. In Proceeedings of the 2017 IEEE 2nd Advanced Information Technology, Electronic and Automation Control Conference (IAEAC), Chongqing, China, 25-26 March 2017; pp. 951-954. [CrossRef]

9. Belyaev, B.; Serzhantov, A.; Tyurnev, V.; Bal'va, Y.; Leksikov, A.; Galeev, R. Implementation of Cross Couplings in Microwave Bandpass Filters. Microw. Opt. Technol. Lett. 2014, 56, 2021-2025. [CrossRef]

10. Hejazi, Z. EM Full-Wave Analysis and Testing of Novel Quasi-Elliptic Microstrip Filters for Ultra Narrowband Filter Design. Prog. Electromagn. Res. 2008, 85, 261-288. [CrossRef]

11. Aristarkhov, G.; Zvezdinov, N. High-Selectivity Single- and Dual-Resonator Microstrip Filters. J. Commun. Technol. Electron. 2017, 62, 916-920. [CrossRef]

12. Menon, S.K.; Donelli, M. Development of a Microwave Sensor for Solid and Liquid Substances Based on Closed Loop Resonator. Sensors 2021, 21, 8506. [CrossRef]

13. Armghan, A. Complementary Metaresonator Sensor with Dual Notch Resonance for Evaluation of Vegetable Oils in C and X Bands. Appl. Sci. 2021, 11, 5734. [CrossRef] 
14. Zarifi, H.; Thundat, T.; Daneshmand, M. High resolution microwave microstrip resonator for sensing applications. Sensors Actuators Phys. 2015, 233, 224-230. [CrossRef]

15. Bogner, A.; Steiner, C.; Walter, S.; Kita, J.; Hagen, G.; Moos, R. Planar Microstrip Ring Resonators for Microwave-Based Gas Sensing: Design Aspects and Initial Transducers for Humidity and Ammonia Sensing. Sensors 2017, 17, 2422. [CrossRef] [PubMed]

16. Morales-Lovera, H.; Olvera-Cervantes, J.; Perez-Ramos, A.; Corona-Chavez, A.; Saavedra, C. Microstrip sensor and methodology for the determination of complex anisotropic permittivity using perturbation techniques. Sci. Rep. 2022, 12, 2205. [CrossRef] [PubMed]

17. Jaber, N.; Ilyas, S.; Shekhah, O.; Eddaoudi, M.; Younis, M. Multimode MEMS Resonator for Simultaneous Sensing of Vapor Concentration and Temperature. IEEE Sensors J. 2018, 18, 10145-10153. [CrossRef]

18. Baghelani, M.; Abbasi, Z.; Daneshmand, M.; Light, P. Non-invasive continuous-time glucose monitoring system using a chipless printable sensor based on split ring microwave resonators. Sci. Rep. 2020, 10, 12980. [CrossRef]

19. Felix, W.; de Oliveira, A.; de Amorim Junior, R.; Nobre, D.; de Oliveira Souto, L.; da V.E. Silva.; Felix, R. Soil Moisture Sensor with a Microstrip Band Stop Filter. In Proceedings of the 2020 IEEE 6th World Forum on Internet of Things (WF-IoT), New Orleans, LA, USA, 15 July 2020; pp. 1-4. [CrossRef]

20. Govind, G.; Akhtar, M. Metamaterial-Inspired Microwave Microfluidic Sensor for Glucose Monitoring in Aqueous Solutions. IEEE Sensors J. 2019, 19, 11900-11907. [CrossRef]

21. Tiwari, N.; Singh, S.; Mondal, D.; Akhtar, M. Flexible biomedical RF sensors to quantify the purity of medical grade glycerol and glucose concentrations. Int. J. Microw. Wirel. Technol. 2020, 12, 120-130. [CrossRef]

Jankovic, N.; Radonic, V. A Microwave Microfluidic Sensor Based on a Dual-Mode Resonator for Dual-Sensing Applications. Sensors 2017, 17, 2713. [CrossRef]

23. Alahnomi, R.A.; Zakaria, Z.; Yussof, Z.M.; Althuwayb, A.A.; Alhegazi, A.; Alsariera, H.; Rahman, N.A. Review of Recent Microwave Planar Resonator-Based Sensors: Techniques of Complex Permittivity Extraction, Applications, Open Challenges and Future Research Directions. Sensors 2021, 21, 2267. [CrossRef] [PubMed]

24. Munoz-Enano, J.; Coromina, J.; Vélez, P.; Su, L.; Gil, M.; Casacuberta, P.; Martín, F. Planar Phase-Variation Microwave Sensors for Material Characterization: A Review and Comparison of Various Approaches. Sensors 2021, 21, 1542. [CrossRef] [PubMed] Podunavac, I.; Radonic, V.; Bengin, V.; Jankovic, N. Microwave Spoof Surface Plasmon Polariton-Based Sensor for Ultrasensitive Detection of Liquid Analyte Dielectric Constant. Sensors 2021, 21, 5477. [CrossRef] [PubMed]

26. Juan, C.; Potelon, B.; Quendo, C.; Bronchalo, E. Microwave Planar Resonant Solutions for Glucose Concentration Sensing: A Systematic Review. Appl. Sci. 2021, 11, 7018. [CrossRef]

27. Qiang, T.; Wang, C.; Kim, N.Y. Quantitative detection of glucose level based on radiofrequency patch biosensor combined with volume-fixed structures. Biosens. Bioelectron. 2017, 98, 357-363. [CrossRef]

28. Jang, C.; Lee, H.J.; Yook, J.G. Radio-Frequency Biosensors for Real-Time and Continuous Glucose Detection. Sensors 2021, 21, 1843. [CrossRef]

29. Yilmaz, T.; Foster, R.; Hao, Y. Radio-Frequency and Microwave Techniques for Non-Invasive Measurement of Blood Glucose Levels. Diagnostics 2019, 9, 6. [CrossRef]

30. Oliveira, J.G.D.; Junior, J.G.D.; Pinto, E.N.M.G.; Neto, V.P.S.; D’Assuncao, A.G. A New Planar Microwave Sensor for Building Materials Complex Permittivity Characterization. Sensors 2020, 20, 6328. [CrossRef]

31. Ansari, M.A.H.; Jha, A.K.; Akhter, Z.; Akhtar, M.J. Multi-Band RF Planar Sensor Using Complementary Split Ring Resonator for Testing of Dielectric Materials. IEEE Sens. J. 2018, 18, 6596-6606. [CrossRef]

32. Tiwari, N.K.; Jha, A.K.; Singh, S.P.; Akhter, Z.; Varshney, P.K.; Akhtar, M.J. Generalized Multimode SIW Cavity-Based Sensor for Retrieval of Complex Permittivity of Materials. IEEE Trans. Microw. Theory Tech. 2018, 66, 3063-3072. [CrossRef]

33. Baltes, H.; Hesse, J.; Gopel, W., Eds. Sensors Update; Wiley-VCH: Hoboken, NJ, USA, 2000; Volume 7.

34. Nyfors, E. Industrial Microwave Sensors-A Review. Subsurf. Sens. Technol. Appl. 2000, 1, 23-43. [CrossRef]

35. Cheng, W.; Deng, K.; Cheng, W. Design of Micro-strip Symmetrical Dual-band Filter Based on Wireless Sensor Network Nodes. EURASIP J. Wirel. Commun. Netw. 2019, 34,1-8. [CrossRef]

36. Liu, W.; Wang, M.; Wu, W. Design on Bi-Directional Circularly Polarized Microstrip Antenna for Mobile Communication J. Antennas 2014, 3, 9-14. [CrossRef]

37. Aleksandrovskij, A.; Belyaev, B.; Leksikov, A. Synthesis and Selective Features of Microstrip Filters Based on Hairpin Resonators with Stub Elements. Radiotekhnika Elektron. 2003, 48, 398-405.

38. Belyaev, B.; Serzhantov, A.; Tyurnev, V. A Dual-Mode Split Microstrip Resonator and its Applications in Frequency Selective Devices. Microw. Opt. Technol. Lett. 2013, 55, 2186-2190. [CrossRef]

39. Belyaev, B.; Khodenkov, S.; Leksikov, A.; Shabanov, V.F. A three-mode microstrip resonator and a miniature ultra-wideband filter based on it. Dokl. Phys. 2017, 62, 289-293. [CrossRef]

40. Chen, C.F.; Lin, C.; Weng, J.H.; Tsai, K.L. Compact microstrip broadband filter using multimode stub-loaded resonator. Electron. Lett. 2013, 49, 545-546. [CrossRef]

41. Djeddi, T.; Elsaadany, M.; Shams, S.; Hamouda, W. Compact Ultra-Wideband Printed Bandpass Filter Based on CoupledLine Resonator Loading. In Proceedings of the 2018 18th International Symposium on Antenna Technology and Applied Electromagnetics (ANTEM), Waterloo, ON, Canada 19-22 August 2018; pp. 1-2. [CrossRef] 
42. Hashemi, S.; Mirshekar-Syahkal, D. Ultra compact filters for ultra-wideband (UWB) applications using multilayer ring resonators. In Proceedings of the 2009 IEEE International Conference on Ultra-Wideband, Vancouver, BC, Canada, 9-11 September 2009; pp. 516-520. [CrossRef]

43. Rahman, M.; NaghshvarianJahromi, M.; Mirjavadi, S.S.; Hamouda, A.M. Resonator Based Switching Technique between Ultra Wide Band (UWB) and Single/Dual Continuously Tunable-Notch Behaviors in UWB Radar for Wireless Vital Signs Monitoring. Sensors 2018, 18, 3330. [CrossRef] [PubMed]

44. Mahmud, M.Z.; Islam, M.T.; Misran, N.; Almutairi, A.F.; Cho, M. Ultra-Wideband (UWB) Antenna Sensor Based Microwave Breast Imaging: A Review. Sensors 2018, 18, 2951. [CrossRef]

45. Shaman, H. Compact microstrip bandpass filter for radars and wireless communication. IETE J. Res. 2013, 59, 496-499. [CrossRef]

46. Yogantana, A.P.; Erfansyah, A. Design of Microstrip Composite Band Pass Filter for Ground Penetrating Radar. Int. J. Adv. Trends Comput. Sci. Eng. 2020, 9, 198-203. [CrossRef]

47. Ouf, E.; Mohra, A.; Abdallah, E.; Elhennawy, Y. A Reconfigurable UWB Bandpass Filters with Embedded Multi-Mode Resonators. Open J. Antennas Propag. 2018, 6, 43-59. [CrossRef]

48. Mahnoor, B.; Najam, A.I. Design Of Compact Wideband Microstrip Filter For Phased Array Radar Applications. In Proceedings of the 2020 17th International Bhurban Conference on Applied Sciences and Technology (IBCAST), Islamabad, Pakistan, 14-18 January 2020; pp. 611-616. [CrossRef]

49. Praludi, T.; Sulaeman, Y.; Taryana, Y.; Sukoco, B.E. Bandpass filter microstrip using octagonal shape for S-band radar. In Proceedings of the 2017 International Conference on Radar, Antenna, Microwave, Electronics, and Telecommunications (ICRAMET), Jakarta, Indonesia 23-24 October 2017; pp. 145-148. [CrossRef]

50. $\mathrm{Xu}, \mathrm{J} . ; \mathrm{Zhu}, \mathrm{Y}$. Compact semi-lumped dual- and tri-wideband band-pass filters. IET Microwaves Antennas Propag. 2017, 11, 53-58. [CrossRef]

51. Dovbysh, I.; Tyurnev, V. Synthesis and Investigation of Three-Section Microstrip Filter on Folded Dual-Mode Stepped-Impedance Resonators. Prog. Electromagn. Res. 2010, 12, 17-28. [CrossRef]

52. Sloss, A.N.; Gustafson, S. 2019 Evolutionary Algorithms Review; GPTP; Springer: Cham, Switzerland, 2019.

53. Stanovov, V.; Akhmedova, S.; Semenkin, E. Genetic Algorithm with Success History Based Parameter Adaptation. In Proceedings of the 11th International Joint Conference on Computational Intelligence (IJCCI 2019), Vienna, Austria, 17-19 September 2019.

54. Bonyadi, M.; Michalewicz, Z. Particle Swarm Optimization for Single Objective Continuous Space Problems: A Review. Evol. Comput. 2017, 25, 1-54. [CrossRef] [PubMed]

55. Das, S.; Suganthan, P. Differential evolution: a survey of the state-of-the-art. IEEE Trans. Evol. Comput. 2011, 15, 4-31. [CrossRef]

56. Kar, A. Bio inspired computing - a review of algorithms and scope of applications. Expert Syst. Appl. 2016, 59, 20-32. [CrossRef]

57. Słowik, A.; Kwasnicka, H. Evolutionary algorithms and their applications to engineering problems. Neural Comput. Appl. 2020, 32, 12363-12379. [CrossRef]

58. Tanabe, R.; Fukunaga, A. Improving the search performance of SHADE using linear population size reduction. In Proceedings of the IEEE Congress on Evolutionary Computation, CEC, Beijing, China, 6-11 July 2014; pp. 1658-1665. [CrossRef]

59. Abalkhail, N.; Alolayan, S.; Albishi, A. Split-Ring Resonator Excited by a U-Shaped Transmission Line for Material Characterization. In Proceedings of the 2020 IEEE International Symposium on Antennas and Propagation and North American Radio Science Meeting, Toronto, ON, Canada, 5-10 July 2020; pp. 981-982. [CrossRef]

60. Zakharov, A.V.; Ilchenko, M.Y.; Pinchuk, L.S. Microstrip U-shaped resonators. Radioelectron. Commun. Syst. 2012, 55, 368-374. [CrossRef]

61. Luo, Y.; Bornemann, J. Open and short U-shaped microstrip resonators for second-order single- or dual-bandstop filter design. Microw. Opt. Technol. Lett. 2017, 59, 1362-1365. doi:10.1002/mop.30544. [CrossRef]

62. Ogbodo, E.A.; Wang, Y.; Yeo, K.S.K. Microstrip Dual-Band Bandpass Filter Using U-Shaped Resonators. Prog. Electromagn. Res. Lett. 2016, 59, 1-6. doi:10.2528/PIERL15072303. [CrossRef]

63. Song, K.; Xue, Q. Novel Broadband Bandpass Filters Using Y-Shaped Dual-Mode Microstrip Resonators. IEEE Microw. Wirel. Components Lett. 2009, 19, 548-550. [CrossRef]

64. Valencia-Ponce, M.A.; Tlelo-Cuautle, E.; de la Fraga, L.G. On the Sizing of CMOS Operational Amplifiers by Applying Many-Objective Optimization Algorithms. Electronics 2021, 10, 3148. [CrossRef]

65. Dor, A.E.; Fakhfakh, M.; Siarry, P. Multiobjective Differential Evolution algorithm using crowding distance for the optimal design of analog circuits. In Proceedings of the 2016 10th International Power Engineering and Optimization Conference (PEOCO2016), Shah Alam, Malaysia, 30 January-5 May 2016; pp. 57-61.

66. Ye, X.; Chen, H.; Kuang, Q.; Wang, Y.; Niu, H.; Li, W.; Yuan, R.; Zhai, G. Solving Time-dependent reliability-based design optimization by adaptive differential evolution algorithm and time-dependent polynomial chaos expansions (ADE-T-PCE). Microelectron. Reliab. 2020, 114, 113815. doi:10.1016/j.microrel.2020.113815. [CrossRef]

67. Kazakovtsev, L.A.; Stupina, A.A. Fast genetic algorithm with greedy heuristic for p-median and k-means problems. In Proceedings of the 2014 6th International Congress on Ultra Modern Telecommunications and Control Systems and Workshops (ICUMT), St. Petersburg, Russia, 6-8 October 2014; pp. 602-606. [CrossRef] 
68. Kazakovtsev, L.; Stashkov, D.; Gudyma, M.; Kazakovtsev, V. Algorithms with greedy heuristic procedures for mixture probability distribution separation. Yugosl. J. Oper. Res. 2019, 29, 51-67. [CrossRef]

69. Storn, R.; Price, K. Differential evolution-A simple and efficient heuristic for global optimization over continuous spaces J. Glob. Optim. 1997, 11, 341-359. [CrossRef]

70. Zhang, R.; Zhang, Y.; Sun, J.; Li, Q. Pattern Synthesis of Linear Antenna Array Using Improved Differential Evolution Algorithm with SPS Framework. Sensors 2020, 20, 5158. [CrossRef]

71. Zhang, J.; Sanderson, A.C. JADE: Adaptive Differential Evolution With Optional External Archive. IEEE Trans. Evol. Comput. 2009, 13, 945-958. [CrossRef]

72. Biedrzycki, R.; Arabas, J.; Jagodzinski, D. Bound constraints handling in Differential Evolution: An experimental study. Swarm Evol. Comput. 2019, 50, 100453. [CrossRef]

73. Tanabe, R.; Fukunaga, A. Success-history based parameter adaptation for differential evolution. In Proceedings of the IEEE Congress on Evolutionary Computation, Cancun, Mexico, 20-23 June 2013; pp. 71-78. [CrossRef]

74. Al-Dabbagh, R.D.; Neri, F.; Idris, N.; Baba, M.S. Algorithmic design issues in adaptive differential evolution schemes: Review and taxonomy. Swarm Evol. Comput. 2018, 43, 284-311. [CrossRef]

75. Deb, K.; Agrawal, R.B. Simulated Binary Crossover for Continuous Search Space. Complex Syst. 1995, 9, 115-148.

76. Kanpur Genetic Algorithms Laboratory; Mechanical Engineering; Technology Kanpur. A Niched-Penalty Approach for Constraint Handling in Genetic Algorithms; Springer: Vienna, Austria, 2002.

77. Deb, K.; Beyer, H.G. Self-Adaptive Genetic Algorithms with Simulated Binary Crossover. Evol. Comput. 2001, 9, 197-221. [CrossRef]

78. Deb, K.; Deb, D. Analysing mutation schemes for real-parameter genetic algorithms. Int. J. Artif. Intell. Soft Comput. 2014, 4, 1-28. [CrossRef]

79. Zhang, Y.; Lu, J.; Le, Z.; Dong, C.H.; Zheng, H.; Qin, Y.; Yu, P.; Hu, W.; Zou, C.L.; Ren, H. Proposal of Unsupervised Gas Classification by Multimode Microresonator. IEEE Photonics J. 2021, 13, 1-11. [CrossRef]

80. Islam, M.T.; Ashraf, F.B.; Alam, T.; Misran, N.; Mat, K.B. A Compact Ultrawideband Antenna Based on Hexagonal Split-Ring Resonator for $\mathrm{pH}$ Sensor Application. Sensors 2018, 18, 2959. [CrossRef] [PubMed]

81. Xu, Y.; Wu, X.; Guo, X.; Kong, B.; Zhang, M.; Qian, X.; Mi, S.; Sun, W. The Boom in 3D-Printed Sensor Technology. Sensors 2017, 17, 1166. [CrossRef] [PubMed]

82. Mohammed, A.M.; Wang, Y.; Lancaster, M.J. 3D printed coaxial microwave resonator sensor for dielectric measurements of liquid. Microw. Opt. Technol. Lett. 2021, 63, 805-810. doi: 10.1002/mop.32679. [CrossRef]

83. Salim, A.; Ghosh, S.; Lim, S. Low-Cost and Lightweight 3D-Printed Split-Ring Resonator for Chemical Sensing Applications. Sensors 2018, 18, 3049. [CrossRef]

84. Han, T.; Kundu, S.; Nag, A.; Xu, Y. 3D Printed Sensors for Biomedical Applications: A Review. Sensors 2019, 19, 1706. [CrossRef]

85. Heger, Z.; Zitka, J.; Cernei, N.; Krizkova, S.; Sztalmachova, M.; Kopel, P.; Masarik, M.; Hodek, P.; Zitka, O.; Adam, V.; Kizek, R. 3D-printed biosensor with poly(dimethylsiloxane) reservoir for magnetic separation and quantum dots-based immunolabeling of metallothionein. Electrophoresis 2015, 36, 1256-1264. [CrossRef]

86. Cevenini, L.; Calabretta, M.M.; Tarantino, G.; Michelini, E.; Roda, A. Smartphone-interfaced 3D printed toxicity biosensor integrating bioluminescent "sentinel cells". Sensors Actuators Chem. 2016, 225, 249-257. [CrossRef]

87. Belyaev, B.; Khodenkov, S.; Galeev, R.G.; Shabanov, V.F. A Lowpass Filter Based on a 2D Microstrip Electromagnetic Crystal. Dokl. Phys. 2019, 64, 85-89. [CrossRef] 